\title{
Multi-Level Cooperative Fusion of GM-PHD Filters for Online Multiple Human Tracking
}

\author{
Zeyu Fu, Member, IEEE, Federico Angelini, Student Member, IEEE, Jonathon Chambers, Fellow, IEEE, and \\ Syed Mohsen Naqvi, Senior Member, IEEE.
}

\begin{abstract}
In this paper, we propose a multi-level cooperative fusion approach to address the online multiple human tracking problem in a Gaussian Mixture Probability Hypothesis Density (GM-PHD) filter framework. The proposed fusion approach consists essentially of three steps. Firstly, we integrate two human detectors with different characteristics (full-body and bodyparts), and investigate their complementary benefits for tracking multiple targets. For each detector domain, we then propose a novel Discriminative Correlation Matching (DCM) model, and fuse it with spatio-temporal information to address ambiguous identity association in the GM-PHD filter. Finally, we develop a robust fusion center with virtual and real zones to make a global decision based on preliminary candidate targets generated by each detector. This center also mitigates the sensitivity of missed detections in the Generalized Covariance Intersection (GCI) fusion process, thereby improving the fusion performance and tracking consistency. Experiments on the MOTChallenge Benchmark demonstrate the proposed method achieves improved performance over other state-of-the-art RFS based tracking methods.
\end{abstract}

Index Terms-Multiple human tracking, GM-PHD filter, data fusion

\section{INTRODUCTION}

$\mathbf{M}$ Ultiple human tracking plays an important part in many applications such as video surveillance, human behavior analysis, and healthcare systems [1]-[6]. Major tasks of multiple human tracking are to accurately retrieve trajectories and maintain target identities given a video sequence [7]. However, there are still challenging issues that hinder the tracking performance, such as background noises, missed detections, and long-term occlusions [5]. To deal with these challenging issues, many researchers have established a wide variety of approaches to overcome this tracking task. With the advancement of object detection, the tracking-by-detection framework has been quite successful to track multiple humans in video [7]-[11]. There are effectively two tracking modes in this framework: online and offline tracking. Offline tracking approaches [8], [10], [12] employ both past and future detections to globally formulate an optimization problem, which is unsuitably applied in real world applications. Online tracking approaches [4], [5], [7], [9], [11], [13] achieve the tracking estimates only relying on detections from past and current time.

Another increasing trend in the tracking-by-detection framework is based on Random Finite Set (RFS) theory [14] and

Z. Fu, F. Angelini, J. Chambers, and S. M. Naqvi are with the Intelligent Sensing and Communications Research Group, School of Engineering, Newcastle University, United Kingdom, E-mails:\{z.fu2, f.angelini2, jonathon.chambers, mohsen.naqvi\}@ newcastle.ac.uk.

E-mail for correspondence: Mohsen.Naqvi@newcastle.ac.uk mainly exploited by the signal processing community. GMPHD and Sequential Monte Carlo (SMC)-PHD filters are two commonly used implementations in this theory, as they have been able to generate convincing tracking performance in video-based multi-target tracking [2], [3], [5], [7], [15][17]. This is attributed to the advantages of PHD filtering methods, as they have the ability to deal with varying number of targets, and also provide the estimates in both cardinality and localization with relatively low computational cost [2]. However, conventional PHD filters are inherently unable to assign identity to targets, so an additional labelling mechanism is needed for completeness, such as the early association in [15] and the post-processing step in [17]. In reality, the performance of these methods may degrade drastically when targets are moving in close proximity, because only relying on target motion is not robust enough to address the target ambiguity in the image plane. Therefore, it is necessary to explore further the visual content to penalize ambiguous targets, which can be achieved by methods from the field of computer vision.

More recently, fusion of multiple data sources has been proven to enhance the tracking robustness and reliability, since this approach can provide redundancy in different aspects, and eliminate uncertainties between individual sources [6], [7], [18]. Fusion based tracking is typically divided in three main categories: detection-level fusion [8], [19], feature-level fusion, and decision-level fusion [6]. Based on the processing level, sequential [14], [20], parallel [21]-[23] and hybrid data fusion [24] approaches are possible. The Generalized Covariance Intersection (GCI) [25] rule was proposed by Mahler [25] for fusion of multi-object functions. It has been demonstrated to effectively fuse multi-target densities with various forms from different sensors with completely unknown correlation [22], [23], [26], [27]. However, established formulations with the conventional GCI rule are prone to missed detections, which may no longer be effective when applied in the video-based tracking task.

In this work, we aim to address the aforementioned issues in PHD filtering via a multi-level cooperative fusion approach for online multiple human tracking. The overview of the proposed tracking approach is illustrated in Fig. 11. The proposed approach is composed of two key ideas: enhanced identity association and a robust fusion center. For the enhanced identity association, we propose a discriminative correlation matching (DCM) model and fuse it with spatio-temporal information to reduce the target ambiguity in the GM-PHD filter. In the matching scheme, we mainly exploit Discriminative 


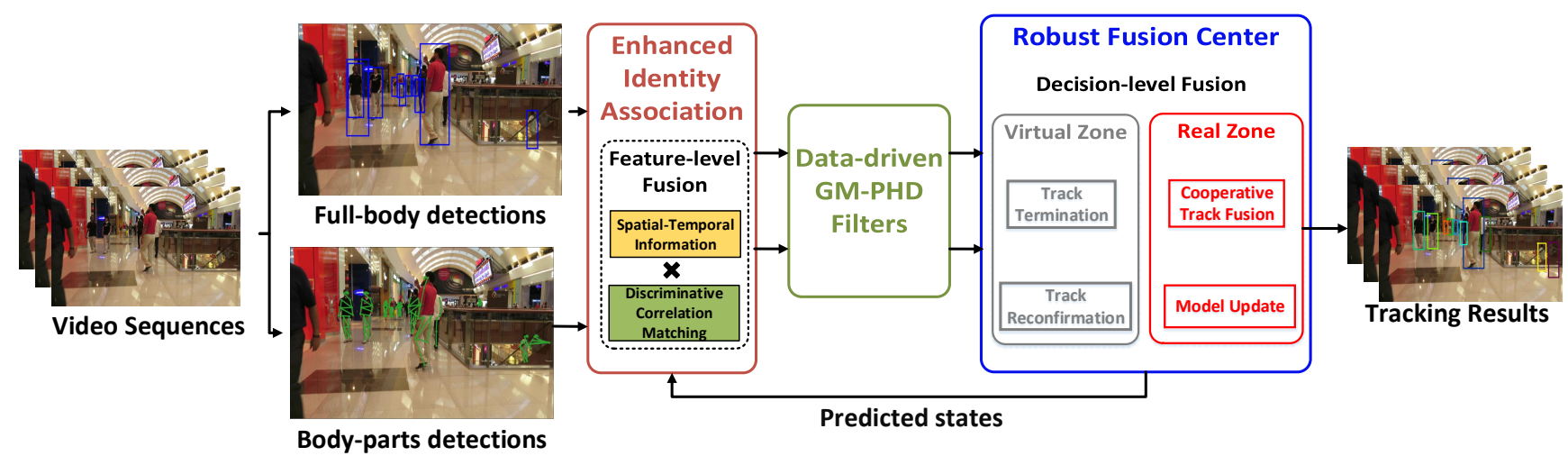

Fig. 1. Overview of the proposed approach for online multiple human tracking. At the first stage, each detector locally achieves valid detections. In the enhanced identity association stage, detections from each domain are locally associated with predicted states by exploiting spatio-temporal information and discriminative correlation matching. The resulting association outputs from each domain are further processed through the GM-PHD filters to achieve the local tracking estimates. Cooperative track fusion and appearance model updates are only performed on the significant tracks in the real zone of the robust fusion center, which yields the final tracking results. The virtual zone in the robust fusion center is designed to manage track termination and reconfirmation.

Correlation Filters (DCFs) learned from features of multiple convolutional layers as target-specific classifiers, which discriminate the desired target from background and other existing targets. Features are obtained from the outputs of both top and lower convolutional layers, which are capable to encode the target appearances with better discriminability of the background and targets in the same category [16], [28]. A robust fusion center is designed with virtual and real zones at the decision level: the real zone is mainly responsible for guiding common tracking estimates of survival and newborn targets collected from both detectors to be fused via the adapted GCI rule; the virtual zone manages to reconstruct the missed targets and remove false detections. The intuition of this design is to enable the fusion process in the tracking system to better explore the maximum strengths from the two detectors, and also mitigates the sensitivity of missed detection occurring in the original GCI rule [7]. Besides, we developed an identity reassignment mechanism similar to [27], to overcome the identity mismatching problem, thereby mitigating the influence of false positives.

The main contributions of our work are summarized as follows:

1) We propose a novel online multiple human tracking method by performing multi-level (feature-level and decision-level) cooperative fusion within the GM-PHD filter framework.

2) A robust fusion center at the decision-level fusion is proposed to improve the fusion process and tracking consistency.

3) A novel DCM model at the feature-level fusion is proposed, which is fused with spatio-temporal information to enhance the ambiguous identity associations in the GM-PHD filter.

4) MOTChallenge Benchmark evaluations are provided to confirm improved performance over other state-of-theart RFS based tracking methods.

Preliminary parts of this work have previously been presented in [7], [16], which were mainly to address the issue of target ambiguity in the GM-PHD filter, as well as justifying the complementary benefits of using two human detectors in multiple human tracking. In this paper, we present the entire multi-level cooperative fusion for the first time. We extend the previous work in [7], [16] to a multi-level cooperative fusion approach with GM-PHD filters, including the integration of multiple measurements using the newly proposed robust fusion center with real and virtual zones to perform cooperative detector fusion. Extensive experiments on MOTChallenge benchmark datasets are conducted to compare the proposed tracking method with recent state-of-the-art trackers. An ablation study on different contribution components and parameter analysis are also included in this work.

\section{RELATED WORK}

In this section, we discuss some recent works on multiple human tracking in three aspects: tracking-by-detection approaches, correlation filter assisted tracking approaches and data fusion approaches.

The major issue of a tracking-by-detection framework is how to perform optimal association of target detections. Existing tracking methods, depending on this framework, can be divided into two modes: online and offline. Online tracking methods achieve the tracking estimates only relying on detections from past and current detections. Sadeghian et al. [9] presented a structure of Recurrent Neural Networks (RNNs) to encode long-term dependencies across motion, appearance and interaction models. Zhou et al. [13] proposed a deep continuous conditional random field with unary and asymmetric pairwise terms to better model the target appearances and inter-object relations. More recently, Chen et al. [11] made full advantage of deep neural networks, which are used to develop a scoring function for candidate selection, as well as adopting person re-identification features for data association. Offline tracking approaches employ both past and future detections to globally formulate an optimization problem. In [29], tracking multiple targets was formulated as a submodular maximization problem to globally find the most related tracklets for trajectory generation. In [12], authors 
exploited the interactions between non-associable tracklets to facilitate multi-target tracking, and addressed the binary labeling problem using efficient quadratic pseudo-Boolean optimization. Kim et al. [30] proposed a novel near online tracking framework. In this framework, they developed a bilinear Long Short-Term Memory (LSTM) which jointly encodes both appearance and motion information for target tracks, in order to make the full use of past target appearances. Since our proposed tracking algorithm focuses on the GM-PHD filtering framework, we also review several recently developed PHD filtering based trackers for multiple human tracking, such as a social force model aided particle PHD filter [2], SMCPHD filter with online group-structured dictionary learning [5], early-association based particle PHD filter [15] and GM-PHD filter with hierarchical association [31]. These methods did not fully address the issues of identity labelling or target ambiguity in the PHD filter. Here we intend to incorporate a robust targetspecific appearance model to mitigate the ambiguous identity association in the filtering process.

Recently, DCFs have been successfully explored in single object tracking applications [32] due to the high computational efficiency. To investigate how correlation filters can improve multiple human tracking, [33] and [34] have been proposed to apply multiple single object trackers based on the Kernelized Correlation Filters (KCFs) in parallel for fast tracking. In [35], authors proposed to integrate correlation filters (CFs) and a confidence-based relative motion network to perform a two-step data association to track multiple objects, where CFs are employed as a verifying step to confirm the target estimates. Furthermore, a recent RFS based tracking approach [20] was proposed to perform the $\mathrm{KCF}$ as an extended step after the PHD update, where the $\mathrm{KCF}$ is mainly used to perform the refinement of target prediction oriented by the label tree technique [36]. However, the above approaches can easily be sensitive to false positives, when CFs are performed with unreliable references or labels [16].

Although the above methods have been able to achieve stateof-the-art performance, these single detector based tracking approaches are limited to exploring the image context more comprehensively, as depicted in Fig. 2. Multi-detector fusion provides an effective solution to reinforce the tracking process. Most existing fusion based tracking methods focus on single level fusion, such as fusing multiple features [37], grouping detections [8], [19] or integrating the tracking outputs [6]. To be more specific, Zhou et al. [37] developed a multi-feature fusion approach, which combines spatial-colour appearance, histogram of oriented gradient and Gaussian spatial constraints to realize the weight penalization, thereby improving the tracking accuracy. In [19] and [8], both methods have well established the idea of grouping detections by modelling the deformable spatial relationship or addressing a weighted graph labelling problem to advance the tracking task. Khalid et al. [6] proposed to fuse a variety of trackers at decision level by hierarchically clustering the trackers based on spatio-temporal agreement to achieve final tracking estimates. Different from the aforementioned fusion based trackers, we present a multilevel cooperative fusion approach that can simultaneously overcome the association ambiguities and improve the tracking
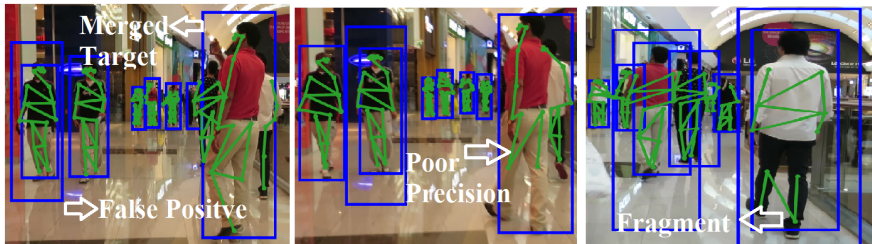

(a)
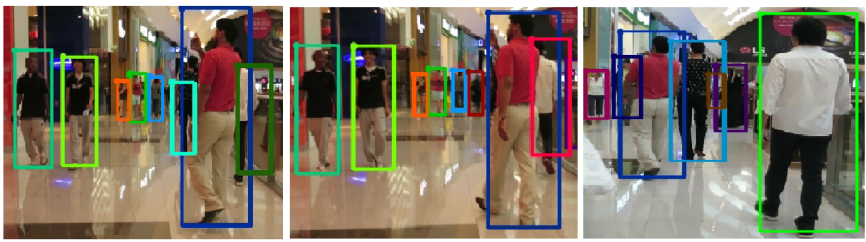

(b)

Fig. 2. Justification of using multiple detectors on the MOT16-11 video sequence [7]. (a) The full-body detector (blue) fails to consider three pedestrains on the right as a merged one when they are in close proximity, and it is prone to false positives. The body-parts detector (green) improves the false positives and merged targets, whilst it has less promising performance in precision and fragmentation. (b) Fusion of both detectors show that false positives are eliminated, missed targets are recovered from the occlusion area, and that tracking precision is improved (better viewed in color version).

reliability. To implement multi-sensor fusion in the PHD filtering framework, two implementations of GCI [25] distributed fusion have been realized in the SMC-CPHD filter [22] and the GM-CPHD filter [23]. In [27], authors have exploited the label space mismatching phenomenon of GCI distributed fusion via labelled RFS filters. However, the aforementioned trackers using the original GCI product rule are prone to missed detections [38]. We therefore propose a robust fusion center with virtual and real zones to select the valid Gaussian components to be applied in the fusion process.

\section{Formulation OF MULTI-TARGET DYNAMICAL AND MEASUREMENT MODEL}

\section{A. Multi-Target RFS Modelling}

Suppose that there are $M_{k}$ targets in an input image frame at time $k$, each target in the state space $\mathcal{X}$ is represented by a six dimensional vector $\mathbf{x}_{k}=\left[p_{x, k}, p_{y, k}, v_{x, k}, v_{y, k}, \theta_{k}, h_{k}\right]^{T}$, where $\left(p_{x, k}, p_{y, k}\right)$ denotes 2D image position, $\left(v_{x, k}, v_{y, k}\right)$ are the horizontal and vertical velocities, and $\left(\theta_{k}, h_{k}\right)$ are the width and height of the bounding box of the target. Note that the target's shape is assumed to be a rectangle. Then, a set of $N_{k}$ target detections are generated, each of which is defined as $\mathbf{z}_{k}=\left[\bar{p}_{x, k}, \bar{p}_{y, k}, \bar{\theta}_{k}, \bar{h}_{k}\right]^{T}$ and typically contains target location and size information in the image plane. Based upon the random finite set (RFS) [14] framework, these target states and measurements can be represented respectively, by two finite sets:

$$
\begin{aligned}
\mathbf{X}_{k} & =\left\{\mathbf{x}_{k, 1}, \ldots, \mathbf{x}_{k, M_{k}}\right\} \in \mathcal{F}(\mathcal{X}) \\
\mathbf{Z}_{k} & =\left\{\mathbf{z}_{k, 1}, \ldots, \mathbf{z}_{k, N_{k}}\right\} \in \mathcal{F}(\mathcal{Z})
\end{aligned}
$$

where $\mathcal{F}(\mathcal{X})$ and $\mathcal{F}(\mathcal{Z})$ are the finite subsets of $\mathcal{X}$ and $\mathcal{Z}$ respectively. The PHD filter [14] based upon the RFS theory, is developed to recursively propagate the first-order moment of the multi-target posterior $p_{k \mid k}\left(\mathbf{X}_{k} \mid \mathbf{Z}_{1: k}\right)$, referred to as the 
intensity function $\nu_{k \mid k}\left(\mathbf{x} \mid \mathbf{Z}_{1: k}\right)$ abbreviated by $\nu_{k \mid k}(\mathbf{x})[5]$, [7]. In this work, we use a practical GM implementation [39] of the PHD recursion to formulate our tracking model.

\section{B. Motion Prediction}

The motion of each target in the surveillance region from time $k-1$ to time $k$ follows a linear Gaussian dynamical model [39],

$$
f_{k \mid k-1}(\mathbf{x} \mid \xi)=\mathcal{N}\left(\mathbf{x} ; \mathbf{F}_{k-1} \xi, \mathbf{Q}_{k-1}\right)
$$

where $\mathbf{F}_{k-1}$ is the state transition matrix which models target propagation, $\mathbf{Q}_{k-1}$ is the process noise covariance, and $\xi$ is the previous state. A posterior intensity $\nu_{k-1}$ in a Gaussian mixture form at time $k-1$ is given as,

$$
\nu_{k-1}(\mathbf{x})=\sum_{j=1}^{J_{k-1}} w_{k-1}^{j} \mathcal{N}\left(\mathbf{x} ; \mathbf{m}_{k-1}^{j}, \mathbf{P}_{k-1}^{j}\right)
$$

where $\mathcal{N}(\cdot ; \mathbf{m}, \mathbf{P})$ denotes a Gaussian component with mean $\mathbf{m}$ and covariance $\mathbf{P}, J_{k-1}$ is the number of Gaussian components at time $k-1$, and $w_{k-1}^{j}$ is the corresponding weight of the $j$-th Gaussian component [7]. Then, a labelling method in [36] is used to manage the target identities, which is to assign a unique label $I_{k-1}^{j}$ as a hidden identity to individual Gaussian components to achieve an identity set $\mathcal{I}_{k-1}=\left\{I_{k-1}^{1}, \ldots, I_{k-1}^{J_{k-1}}\right\}|7|$.

For the current time step $k$, we perform the prediction independently, for each individual target survived from previous time. The survival prediction is given by [39],

$$
\begin{gathered}
\nu_{k \mid k-1, S}(\mathbf{x})=e_{k \mid k-1} \sum_{j=1}^{J_{k-1}} w_{k-1}^{j} \mathcal{N}\left(\mathbf{x} ; \mathbf{m}_{k \mid k-1, S}^{j}, \mathbf{P}_{k \mid k-1, S}^{j}\right) \\
\mathbf{m}_{k \mid k-1, S}^{j}=\mathbf{F}_{k-1} \mathbf{m}_{k-1}^{j} \\
\mathbf{P}_{k \mid k-1, S}^{j}=\mathbf{Q}_{k-1}+\mathbf{F}_{k-1} \mathbf{P}_{k-1}^{j}\left(\mathbf{F}_{k-1}\right)^{T}
\end{gathered}
$$

where $\nu_{k \mid k-1, S}(\mathbf{x})$ represents the predicted intensity of survival targets, and $e_{k \mid k-1}$ is the survival probability. The identities of Gaussian components remain unaltered during the prediction, $I_{k \mid k-1, S}^{j}=I_{k-1}^{j}, j=1, \ldots, J_{k-1}$. In reality, these predicted Gaussian terms may contain ghost targets those are potentially missed at current time $k$, which increases the uncertainty in the update step. Therefore, it is required and necessary to examine the predictions in the early stage before implementing the filtering process.

\section{Measurement Model}

To build a robust measurement model, we integrate two sets of measurements (full-body and body-parts detections) per time step $k$ for parallel processing, as illustrated in Fig. 1 We firstly apply a full body detector [40] to form a set of full-body detections $\mathbf{Z}_{k, a}$, where $a$ represents the fullbody detector. Furthermore, it is likely that each person can have multiple measurements that are spatially surrounding the target for each time step, which means that each human target consists of a certain number of body parts that share common dynamics or attributes [7], [41]. To this end, we adopt a body parts detector [42] to acquire body-parts detections $\mathbf{Z}_{k, b}$, where $b$ demonstrates the body-parts detector. There are ideally 14 body parts which can be obtained per time step $k$, namely ankles, knees, hips, wrists, elbows, shoulders with left/right symmetry, and the head top/bottom [7]. A rectangle shape is considered for grouping the body-parts measurements to model the human contour. In this work, we discard the reshaped measurements if their number of body-parts is less than 6 . For simplicity, we denote $\mathbf{Z}_{k, \theta}, \theta \in\{a, b\}$ concisely as $\mathbf{Z}_{k}$ and ignore the obtained detections on the detector index $\theta$. The measurement model for each detection under the Gaussian assumption is given by,

$$
g_{k}(\mathbf{z} \mid \mathbf{x})=\mathcal{N}\left(\mathbf{z}_{k} ; \mathbf{H}_{k} \mathbf{x}_{k}, \mathbf{R}_{k}\right)
$$

where $\mathbf{H}_{k}$ denotes the observation matrix, $\mathbf{R}_{k}$ denotes the observation noise covariance matrix at time $k$. In fact, noisy measurements can degrade the efficiency of the data-driven PHD filter and birth prediction [16]. We carry out a preprocessing on the original detections before performing target association. We use the confidence score $c_{k} \in[0,1]$ built in each full-body detection to remove the noises $\boldsymbol{\Gamma}_{k}=\left\{\mathbf{z}_{k}^{f}\right.$ : $\left.c_{k}<c_{t h}\right\}$ in the original detections $\mathbf{Z}_{k}$, where $c_{t h}$ is the threshold value [16], thus reliable measurements are obtained as, $\mathbf{Z}_{k}^{+}=\mathbf{Z}_{k} \backslash \boldsymbol{\Gamma}_{k}$.

\section{ENHANCED IDENTITY Association}

This section presents an enhanced labelling system for the PHD filtering process. Unlike the early association in [15], our approach integrates DCM appearance models with spatiotemporal information to address the target ambiguity in the labelling system.

\section{A. Spatio-Temporal Information}

Spatio-temporal information has been widely used for measuring affinity between detections and targets [15], [16], [36]. It is able to capture geometric relations between the bounding boxes with low computational cost. The means of Gaussian terms given by (6) are regarded as predicted states of individual targets for the calculation of association cost. Reliable measurements $\mathbf{Z}_{k}^{+}$are previously obtained in Section III-C Then, the association cost $\Lambda_{k}^{S T}$ is measured by spatio-temporal relation between a reliable detection $\mathbf{z}_{k} \in \mathbf{Z}_{k}^{+}$and a predicted state $\mathbf{m}_{k \mid k-1, S}$, which is computed by,

$$
\Lambda_{k}^{S T}\left(\mathbf{m}_{k \mid k-1, S}, \mathbf{z}_{k}\right)=\exp \left(-\frac{\left\|\mathbf{H}_{k} \mathbf{m}_{k \mid k-1, S}-\mathbf{z}_{k}\right\|}{2 \sigma_{s}^{2}}\right)
$$

where $\|\cdot\|$ denotes the Euclidean distance which calculates the relation on the position and size terms, and $\sigma_{s}^{2}$ represents the variance. These affinity scores $\Lambda_{k}^{S T}$ obtained from the spatiotemporal relation construct a cost matrix $\Delta_{k}^{S T}$. However, only acquiring the geometric relations may generate ambiguous affinity scores when targets are in close proximity. To this end, we will explore target relations on the visual content in the next section.

\section{B. Discriminative Correlation Matching}

Recently, discriminative correlation filters have been widely used in single object visual tracking for better accuracy and efficiency. The DCF based tracking approaches that learn 

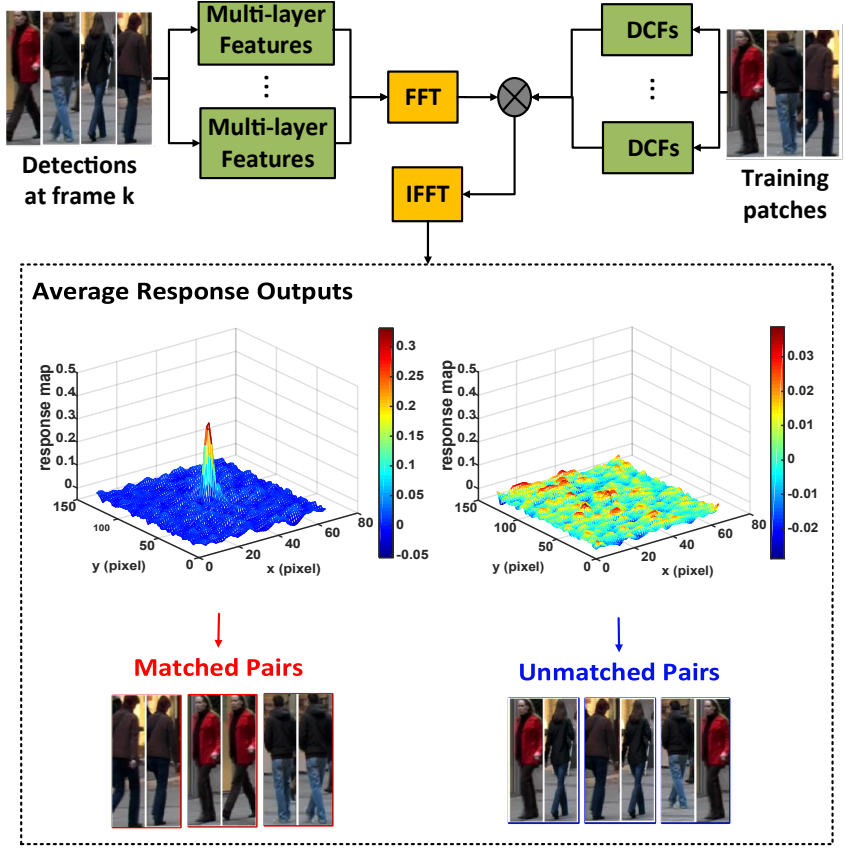

Fig. 3. An example workflow of proposed discriminative correlation matching scheme which uses DCF-based target-specific classifiers with the average response outputs for target appearance matching.

correlation filters to encode target appearances can achieve high computational efficiency [32] with the use of fast Fourier transforms (FFTs). For visual tracking, DCFs are mainly learned as linear classifiers to discriminate between target and background appearances. In this work, we exploit the discriminative power of DCFs and propose a discriminative correlation matching scheme to formulate the appearance model. The major task is to learn the discriminative correlation filters with features of multiple convolutional layers as targetspecific classifiers to discriminate the desired target from noisy background and also other intra-class targets. Preliminary idea has been appeared in our previous conference paper [16]. Fig. 3 shows the workflow of the proposed discriminative correlation matching. For each target, we learn two DCFs on the outputs from both top and lower convolutional layers to encode the target appearances, one (top layer) is used to distinguish between targets and background, the other (lower layer) is for handling intra-class targets variations. We measure the affinity scores by computing the average Peak-to-Sidelobe Ratios (PSRs) inferred from the correlation responses to determine the resulting outputs: matched or unmatched pairs.

1) Training Phase: There are two discriminative correlation filters $\left\{\mathbf{c}^{(l)}\right\}$ trained for each predicted target. The intuition of applying two filters together is to help match between a predicted target and a new detection with the exploitation in both semantic and spatial details of target appearances. Feature maps used for training are from the outputs of both the top and lower convolutional layers, each of which $\mathbf{f}^{(l)}$ of size $A \times B \times D$ are extracted from the output of the $l$ th convolutional layer, where $A, B$, and $D$ denote the width, height, and the number of channels, respectively [16]. Training samples for discriminative correlation filters are generated from all circular shifts $\mathbf{f}_{a, b}^{(l)},(a, b) \in\{0, \ldots, A-1\} \times\{0, \ldots, B-$ $1\}$. Each shifted sample has a desired output $g^{(l)}(a, b)=$ $\exp \left(-\frac{(a-A / 2)^{2}+(b-B / 2)^{2}}{2 \sigma^{2}}\right)$ to form a Gaussian label matrix $\mathbf{g}^{(l)}=\left\{g^{(l)}(a, b) \mid(a, b) \in\{0, \ldots, A-1\} \times\{0, \ldots, B-1\}\right.$, where $\sigma_{c}$ is the kernel width [16]. The DCF $\mathbf{c}^{(l)}$ with the same size of $\mathbf{f}^{(l)}$ can be learned by minimizing the following loss [16], [32],

$$
\underset{\mathbf{c}^{(l)}}{\arg \min } \sum_{a, b}\left\|\sum_{d=1}^{D}\left(\mathbf{c}_{a, b, d}^{(l)}\right)^{T} \mathbf{f}_{a, b, d}^{(l)}-g^{(l)}(a, b)\right\|^{2}+\lambda\left\|\mathbf{c}^{(l)}\right\|_{2}^{2}
$$

where $\lambda$ is the regularization parameter. We follow the literature on training the DCF in [32], which is to perform the fast Fourier transform (FFT) and thereby operate in the frequency domain. Therefore, the solution of $(10)$ on the $d$-th $(d \in 1, \ldots, D)$ channel can be written as [16], [32],

$$
\hat{\mathbf{c}}_{d}^{(l)}=\frac{\hat{\mathbf{g}}^{(l)} \odot\left(\hat{\mathbf{f}}_{d}^{(l)}\right)^{\dagger}}{\sum_{d=1}^{D} \hat{\mathbf{f}}_{d}^{(l)} \odot\left(\hat{\mathbf{f}}_{d}^{(l)}\right)^{\dagger}+\lambda}
$$

where the hat stands for FFT operator, and the dagger represents complex conjugation operation. The operator $\odot$ defines the Hadamard (element-wise) product.

2) Correlation Matching: Prior to performing the matching scheme, it is essential for a newly detected target to extract each feature map $\mathbf{y}^{(l)} \in \mathbb{R}^{A \times B \times D}$ by using the same layers in the training phase. The goal of performing correlation matching in a many-to-many scenario is to find all the correlation responses between each predicted and newly detected target. In practice, each correlation filter $\mathbf{c}^{(l)}$ is correspondingly associated with each feature map $\mathbf{y}^{(l)}$ to compute a correlation response map $\mathbf{r}^{(l)} \in \mathbb{R}^{A \times B}$ at the $l$-th layer [16], [32],

$$
\mathbf{r}^{(l)}=\mathscr{F}^{-1}\left\{\sum_{d=1}^{D} \hat{\mathbf{c}}_{d}^{(l)} \odot\left(\hat{\mathbf{y}}_{d}^{(l)}\right)^{\dagger}\right\}
$$

where $\mathscr{F}^{-1}\{\cdot\}$ denotes the inverse fast Fourier transform (IFFT). Given the set of response maps $\left\{\mathbf{r}^{(l)}\right\}$, we utilize PSRs to obtain the set of scores $\left\{P S R^{(l)}\right\}$ from each layer $l$ :

$$
P S R^{(l)}=\frac{\max \left(\mathbf{r}^{(l)}\right)-\mu^{(l)}}{\sigma_{r}^{(l)}}
$$

where $\mu^{(l)}$ and $\sigma_{r}^{(l)}$ denote the mean value and the standard deviation of the sidelobes. Similar works [35] and [20] used the PSRs as a gating technique to confirm the predicted state or detect tracking failures. However, our work focuses on enhancing the association step. We firstly propose to compute the average sum of the $\left\{P S R^{(l)}\right\}$ to achieve the overall matching score $\rho$. We consider $\rho<\varpi$ as unmatched pairs and $\rho \geq \varpi$ as matched pairs, where the value of the matching threshold $\varpi$ is experimentally chosen in Table III Then a generalized sigmoid function is utilized to compute affinity scores between each predicted and newly detected target based on the correlation matching results, since this function limits the overall score $\rho$ to a range of $[0,1]$. Each pairwise affinity score $\Lambda_{k}^{A}$ is calculated based on an overall matching score $\rho$,

$$
\Lambda_{k}^{A}=\frac{1}{1+e^{-(\alpha \times \rho+\beta)}}
$$

where $\alpha$ and $\beta$ are the coefficients for the calculation. These affinity scores $\Lambda_{k}^{A}$ obtained from the matching scheme construct a cost matrix $\Delta_{k}^{A}$, which is ultimately fused with 
the cost of spatio-temporal relation $\Delta_{k}^{S T}$ to build the total association cost as follows:

$$
\boldsymbol{\Delta}_{k}=\boldsymbol{\Delta}_{k}^{S T} \odot \boldsymbol{\Delta}_{k}^{A}
$$

The benefit of this feature-level fusion is that it can compensate for unreliability present in the individual association cost, especially when target ambiguities occur in either motion dynamics or visual content [16]. In practice, each set of valid detections is locally processed in the enhanced identity association. We use the Hungarian algorithm [43] to achieve the optimal association. The overall algorithm of enhanced identity association is given with details in Algorithm 1 .

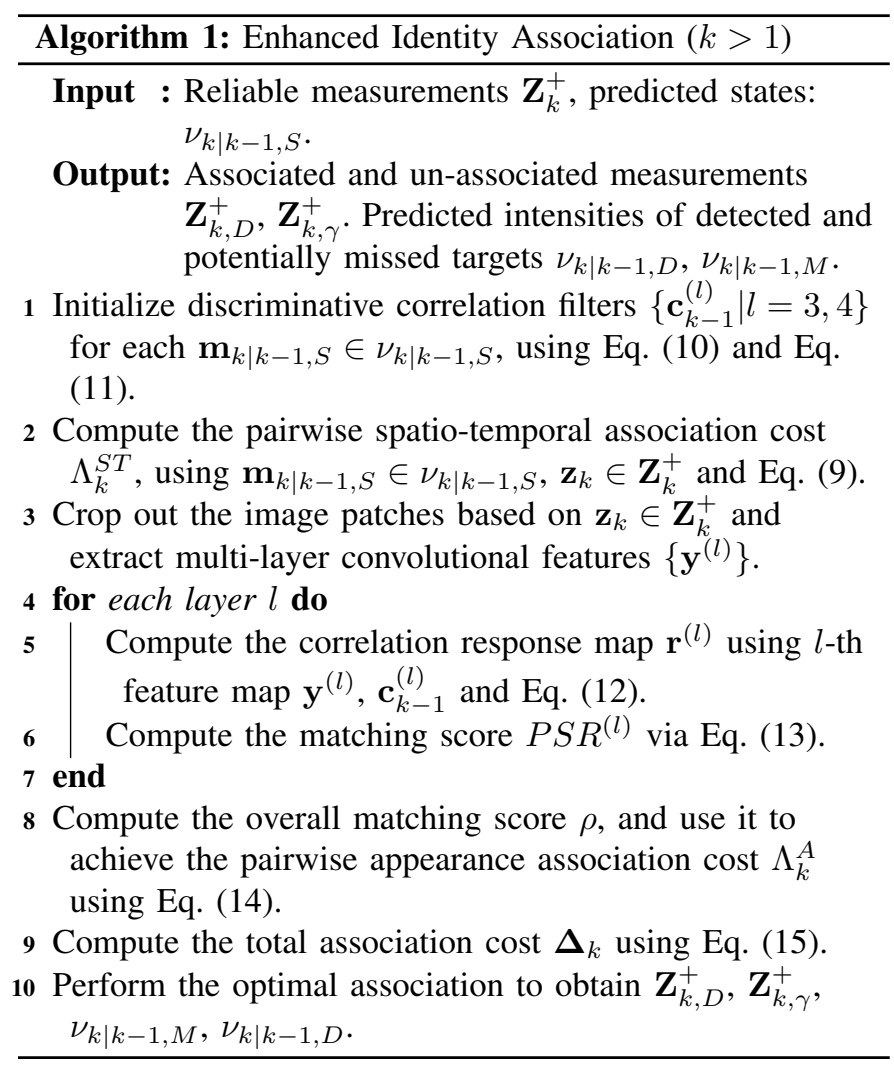

\section{DATA-Driven Gaussian Mixture PHD Filter}

In this section, we present a practical version of the datadriven GM-PHD filter to track multiple humans in video. The filtering process is applied on each set of association results from both detectors to achieve candidate tracking estimates. In the traditional PHD filter, all the input measurements in $\mathbf{Z}_{k}$ are used for the update steps. However, it may degrade the updating performance because of the misuse of measurements and false alarms. For our tracking task, taking advantage of association results in the previous section, the reliable measurement set $\mathbf{Z}_{k}^{+}$achieved in Section III-C can be categorized into,

$$
\mathbf{Z}_{k}^{+}=\mathbf{Z}_{k, D}^{+} \cup \mathbf{Z}_{k, \gamma}^{+}
$$

where $\mathbf{Z}_{k, D}^{+}$and $\mathbf{Z}_{k, \gamma}^{+}$denote the associated and un-associated measurements, respectively. The clutter measurement set $\boldsymbol{\Gamma}_{k}$ which often misleads the tracking process is not included in the update step. Therefore, the PHD filtering process for newborn and survival targets can be performed independently with the corresponding measurements. Associated measurements are only considered for the update of survival targets, while unassociated measurements are used for the target initialization.

\section{A. Target Survival}

Owing to the enhanced identity association, the predicted intensity of survival targets can be reformulated as,

$$
\nu_{k \mid k-1, S}(\mathbf{x})=\nu_{k \mid k-1, M}(\mathbf{x})+\nu_{k \mid k-1, D}(\mathbf{x})
$$

where $\nu_{k \mid k-1, M}(\mathbf{x})$ and $\nu_{k \mid k-1, D}(\mathbf{x})$ are the predicted intensities of potentially missed and detected targets, respectively. In the meanwhile, the identity set of $\nu_{k \mid k-1, S}(\mathbf{x})$ is modified as, $\mathcal{I}_{k \mid k-1, S}=\mathcal{I}_{k \mid k-1, M} \cup \mathcal{I}_{k \mid k-1, D}$. For the update of predicted intensity of potentially missed targets $\nu_{k \mid k-1, M}(\mathbf{x})$, target states and covariance matrices are effectively inherited from the prediction, while weights are modified with the missing detection probability $p_{M}$,

$$
\begin{gathered}
\nu_{k, M}(\mathbf{x})=\sum_{j=1}^{J_{k \mid k-1, M}} w_{k, M}^{j} \mathcal{N}\left(\mathbf{x} ; \mathbf{m}_{k \mid k, M}^{j}, \mathbf{P}_{k \mid k, M}^{j}\right) \\
w_{k, M}^{j}=P_{M} w_{k \mid k-1, M}^{j} \\
\mathbf{m}_{k \mid k, M}^{j}=\mathbf{m}_{k \mid k-1, M}^{j}, \mathbf{P}_{k \mid k, M}^{j}=\mathbf{P}_{k \mid k-1, M}^{j}
\end{gathered}
$$

In addition, the identity set of $\nu_{k, M}(\mathbf{x})$ remains as, $\mathcal{I}_{k \mid k, M}=$ $\mathcal{I}_{k \mid k-1, M}$. The updated intensities of potentially disappearing targets $\nu_{k, M}(\mathbf{x})$ are finally moved into the virtual zone of the proposed fusion center for target termination or reconfirmation.

On the other hand, we employ the associated measurements $\mathbf{Z}_{k, D}^{+}$computed in 16 to update the predicted intensity of detected targets $\nu_{k \mid k-1, D}(\mathbf{x})$ [44],

$$
\nu_{k, D}(\mathbf{x})=\sum_{\mathbf{z} \in \mathbf{Z}_{k, D}^{+}} \sum_{j=1}^{J_{k \mid k-1, D}} w_{k, D}^{j}(\mathbf{z}) \mathcal{N}\left(\mathbf{x} ; \mathbf{m}_{k \mid k, D}^{j}(\mathbf{z}), \mathbf{P}_{k \mid k, D}^{j}\right)
$$

where

$$
\begin{gathered}
w_{k, D}^{j}(\mathbf{z})=\frac{\left(1-p_{M}\right) w_{k \mid k-1, D}^{j} q_{k, D}^{j}(\mathbf{z})}{\kappa_{k}(\mathbf{z})+\left(1-p_{M}\right) \sum_{i=1}^{J_{k \mid k-1, D}} w_{k \mid k-1, D}^{i} q_{k, D}^{i}(\mathbf{z})} \\
q_{k, D}^{j}(\mathbf{z})=\mathcal{N}\left(\mathbf{z} ; \mathbf{H}_{k} \mathbf{m}_{k \mid k-1, D}^{j}, \mathbf{R}_{k}+\mathbf{H}_{k} \mathbf{P}_{k \mid k-1, D}^{j} \mathbf{H}_{k}^{T}\right) \\
\mathbf{m}_{k \mid k, D}^{j}(\mathbf{z})=\mathbf{m}_{k \mid k-1, D}^{j}+\mathbf{K}_{k, D}^{j}\left(\mathbf{z}-\mathbf{H}_{k} \mathbf{m}_{k \mid k-1, D}^{j}\right) \\
\mathbf{P}_{k \mid k, D}^{j}=\left[\mathbf{I}-\mathbf{K}_{k, D}^{j} \mathbf{H}_{k}\right] \mathbf{P}_{k \mid k-1, D}^{j} \\
\mathbf{K}_{k, D}^{j}=\mathbf{P}_{k \mid k-1, D}^{j}\left(\mathbf{H}_{k}\right)^{T}\left(\mathbf{H}_{k} \mathbf{P}_{k \mid k-1, D}^{j}\left(\mathbf{H}_{k}\right)^{T}+\mathbf{R}_{k}\right)^{-1}
\end{gathered}
$$

where $\kappa_{k}$ is the clutter density. Each predicted Gaussian component increases to $\left|\mathbf{Z}_{k, D}^{+}\right|$updated components labelled with the same identity, i.e., $I_{k \mid k, D}^{j}=I_{k \mid k-1, D}^{j}$ [7|. Then, we use the strategy in [36] that selects updated components with the maximum weights as a set of possible estimated states $\tilde{\nu}_{k, D}(\mathbf{x})$ which are taken as inputs to the real zone of the fusion center for the survival fusion process. 


\section{B. Target Initialization}

Standard formulation of PHD filtering methods [39] [45] often presets the target birth model to cover the entire region of interest. In the video tracking context, we rely on the detections from the object detector to handle the target birth and death that are highly random and unpredictable, so as to avoid the need for prior knowledge of the scene information. Therefore, newborn targets will be adaptively estimated by using measurements in $\mathbf{Z}_{k, \gamma}^{+}$,

$$
\begin{gathered}
\gamma_{k}(\mathbf{x})=\sum_{j=1}^{J_{k, \gamma}} w_{k, \gamma}^{j} \mathcal{N}\left(\mathbf{x} ; \mathbf{m}_{k, \gamma}^{j}, \mathbf{P}_{k, \gamma}^{j}\right) \\
\mathbf{m}_{k, \gamma}^{j}=\mathbf{H}_{k}^{-1} \mathbf{z}_{k}^{j} \\
\mathbf{P}_{k, \gamma}^{j}=\mathbf{H}_{k}^{-1} \mathbf{R}_{k}\left(\mathbf{H}_{k}^{-1}\right)^{T}
\end{gathered}
$$

These newborn targets are labelled with new identities $\mathcal{I}_{k, \gamma}=$ $\left\{I_{k, \gamma}^{1}, \ldots, I_{k, \gamma}^{J_{k, \gamma}}\right\}$. where $J_{k, \gamma}=\left|\mathbf{Z}_{k, \gamma}^{+}\right|$denotes the number of new-born targets. According to [44], newborn targets that are adaptively initialized from measurements can be considered to be always detected. Hence, the missed detection probability is always zero $\left(P_{M}=0\right)$ for the update step of newborn targets. Then, the GM-PHD update step for the newly initialized targets is given by,

$$
\nu_{k, \gamma}(\mathbf{x})=\sum_{\mathbf{z} \in \mathbf{Z}_{k, \gamma}^{+}} \sum_{j=1}^{J_{k, \gamma}} w_{k \mid k, \gamma}^{j}(\mathbf{z}) \mathcal{N}\left(\mathbf{x} ; \mathbf{m}_{k \mid k, \gamma}^{j}(\mathbf{z}), \mathbf{P}_{k \mid k, \gamma}^{j}\right)
$$

where $\mathbf{m}_{k \mid k, \gamma}^{j}, \mathbf{P}_{k \mid k, \gamma}^{j}$, and $w_{k \mid k, \gamma}^{j}$ can be achieved by the similar reasoning as in the target survival of (22)-(26). Then, we also adopt the management scheme in [36] to extract the significant components to form $\tilde{\nu}_{k, \gamma}(\mathbf{x})$, which are taken as inputs to the real zone of the fusion center for the birth fusion process.

\section{Robust Fusion CENTER}

After parallel processing from data-driven GM-PHD filters, preliminary tracking estimates which implicitly include the target bounding boxes from each detector domain are passed to a fusion center, where a global decision is performed. The overview of the proposed robust fusion center is depict in Fig. 4

\section{A. Real Zone}

In the real zone, the major processes as shown in Fig. 4 can be divided into two stages: cooperative track fusion and model update. The proposed cooperative track fusion follows the data-driven scheme, which performs survival and birth track fusion independently on the tracking estimates of survival and new-born targets. The overall cooperative track fusion algorithm is given with details in Algorithm 2 . Model update is only performed on the fused survival tracks to deal with the appearance variations.

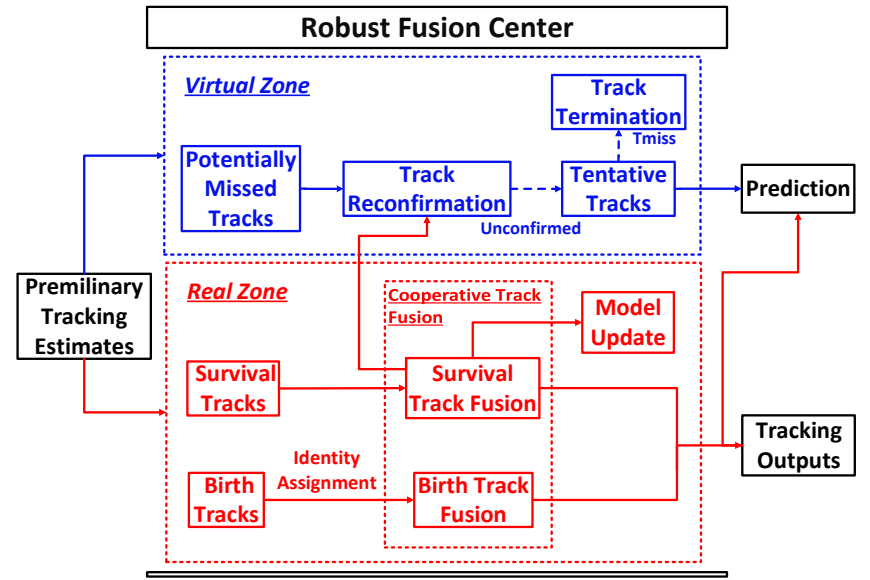

Fig. 4. Overview of the robust fusion center. Real zone (red): the cooperative track fusion is performed, which applies survival and birth track fusion independently on the survival and birth tracks. An identity reassignment mechanism prior to the birth track fusion process is performed to overcome the identity mismatching issue. Model update is only performed on the fused survival tracks to deal with the appearance variations. Virtual zone (blue): potentially missed tracks require further reconfirmation by communicating the non-fused survival tracks from the real zone. Tracks yet reconfirmed are considered as tentative tracks. Track termination is performed to eliminate the tentative tracks with a threshold $T_{m i s s}$. Finally, tentative tracks still remaining in the virtual zone are not added to the final tracks, but are used for prediction in the next time step.

1) Cooperative Track Fusion: In this context, fusing tracks are actually to communicate the GM-PHD intensities, so we exploit the GCI rule which has been widely used in multisensor fusion with the PHD filter. The GCI fusion rule was proposed by Mahler [25] for fusion of multi-object functions, providing a suboptimal solution to preserve maximal information in the fused posterior from local posteriors. Battistelli et al. [23] employed exponential mixture densities (EMD), specifically to realize the GCI fusion of GM-PHD intensities. The GCI fusion rule provides an effective solution to fuse two Gaussian mixtures $v_{a}$ and $v_{b}$. It outputs a fused intensity $\nu_{\varphi}(\mathbf{x})$ with a fusing parameter $0 \leq \varphi \leq 1$ :

$$
\nu_{\varphi}(\mathbf{x})=\frac{\nu_{a}^{\varphi}(\mathbf{x}) \nu_{b}^{1-\varphi}(\mathbf{x})}{\int \nu_{a}^{\varphi}(\mathbf{x}) \nu_{b}^{1-\varphi}(\mathbf{x}) d \mathbf{x}}
$$

Using the Exponential Gaussian Mixture, the power of a Gaussian mixture model can be formulated by the following approximation [23],

$$
\left(\sum_{j=1}^{J} w^{j} \mathcal{N}\left(\mathbf{x} ; \mathbf{m}^{j}, \mathbf{P}^{j}\right)\right)^{\varphi} \approx \sum_{j=1}^{J}\left(w^{j}\right)^{\varphi} \epsilon\left(\varphi, \mathbf{P}^{j}\right) \mathcal{N}\left(\mathbf{x} ; \mathbf{m}^{j}, \frac{\mathbf{P}^{j}}{\varphi}\right)
$$

where

$$
\epsilon\left(\varphi, \mathbf{P}^{j}\right) \triangleq \frac{\left[\operatorname{det}\left(2 \pi \mathbf{P}^{j} \varphi^{-1}\right)\right]^{\frac{1}{2}}}{\left[\operatorname{det}\left(2 \pi \mathbf{P}^{j}\right)\right]^{\frac{\varphi}{2}}}
$$

Therefore, the GCI fusion for GM-PHD intensities can be equivalent to applying Covariance Intersection (CI) pairwise to Gaussian components from two intensities [7]. Both human detectors used in this tracking scenario have fully overlapped field of views (FOVs), which results in applying the GCI fusion rule in our fusion process without requiring transformation. 


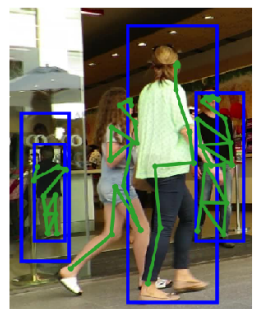

Detections

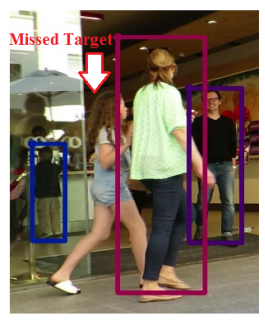

GCI-Fusion

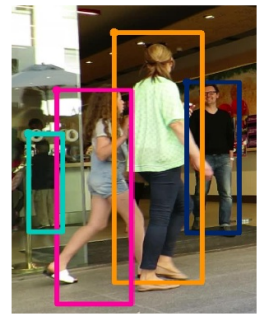

Proposed
Fig. 5. Qualitative comparison between the use of the original GCI fusion and the proposed fusion center on the MOT16-09 video sequence. Detection results show that a target located in the middle of the scene is detected by the body-parts detector (green) but missed by the full-body detector (blue). In this case, fusion through the original GCI rule would lose the target even though it has been detected by the body-parts detector. In the proposed fusion center, this target only observed by the body-parts detector would be reconfirmed and preserved in the tracking outputs (better viewed in color version).

However, a recent study in [38] has suggested that the original GCI fusion rule is prone to missed detections. Missing targets at one detector can degrade the fusion performance with detected targets from other detectors. To be more specific, suppose a target $\mathbf{x}_{k}$ at time $k$ is detected by detector $a$, but missed in detector $b$, then we have $\nu_{a}\left(\mathbf{x}_{k}\right)>0$ and $\nu_{b}\left(\mathbf{x}_{k}\right) \approx 0$. After applying the GCI fusion in $(31)$, the fused result can be $\nu_{\varphi}\left(\mathbf{x}_{k}\right) \approx 0$, which implies that the target is lost even though a larger fusion weight $\varphi$ is given in $\nu_{b}\left(\mathbf{x}_{k}\right)$. We develop a robust fusion center with real and virtual zones to overcome the aforementioned issue, and thus improved the fusion process and tracking consistency. Qualitative results which demonstrate the advantages of the proposed fusion algorithm over the original GCI rule are shown in Fig. 5.

a) Survival Track Fusion: For the survival track fusion, survival tracks from both detectors possess the same identity library. It means that a survival target processed by both detectors will be given by the same identity. Therefore, it is feasible to directly apply CI on survival tracks with the same identities.

Given each pair of Gaussian components $i$ and $j$ with the same label from the intensities $\tilde{\nu}_{k, D}^{a}(\mathbf{x})$ and $\tilde{\nu}_{k, D}^{b}(\mathbf{x})$, each fused component with the corresponding weight can be reformulated with the following characteristics [46],

$$
\begin{gathered}
\mathbf{m}_{a b, k}^{i j}=\mathbf{P}_{a b, k}^{i j}\left[\varphi\left(\mathbf{P}_{a, k}^{i}\right)^{-1} \mathbf{m}_{a, k}^{i}+(1-\varphi)\left(\mathbf{P}_{b, k}^{j}\right)^{-1} \mathbf{m}_{b, k}^{j}\right] \\
\mathbf{P}_{a b, k}^{i j}=\left[\varphi\left(\mathbf{P}_{a, k}^{i}\right)^{-1}+(1-\varphi)\left(\mathbf{P}_{b, k}^{j}\right)^{-1}\right]^{-1} \\
w_{a b, k}^{i j}=\left(w_{a, k}^{i}\right)^{\varphi}\left(w_{b, k}^{j}\right)^{1-\varphi} \epsilon\left(\varphi, \mathbf{P}_{a, k}^{i}\right) \epsilon\left(1-\varphi, \mathbf{P}_{b, k}^{j}\right) \\
\mathcal{N}\left(\mathbf{m}_{a, k}^{i}-\mathbf{m}_{b, k}^{j} ; 0, \frac{\mathbf{P}_{a, k}^{i}}{\varphi}+\frac{\mathbf{P}_{b, k}^{j}}{1-\varphi}\right) \\
\epsilon\left(\varphi, \mathbf{P}_{a, k}^{i}\right)=\frac{\left[\operatorname{det}\left(2 \pi \mathbf{P}_{a, k}^{i} \varphi^{-1}\right)\right]^{\frac{1}{2}}}{\left[\operatorname{det}\left(2 \pi \mathbf{P}_{a, k}^{i}\right)\right]^{\frac{\varphi}{2}}}
\end{gathered}
$$

therefore, these fused components establish the fused intensity of survival tracks $\tilde{\nu}_{k, D}^{a b}(\mathbf{x})$. Conventional fusion approaches [22], [23], [27] usually preset the value of $\varphi$ as 0.5 with the assumption that both sensors have the same sensing abilities in all aspects. However, the aforementioned design of fusion weight may be no longer applicable to real tracking applications, since it is not always feasible for detectors to have the same sensing abilities due to different imaging conditions or camera motions. To this end, we experimentally determine an appropriate value for parameter $\varphi$ in Fig. 7, to handle the trade off between improving the accuracy of MOTA and the MOTP. Non-fused survival tracks from both detectors are preserved into final tracking set.

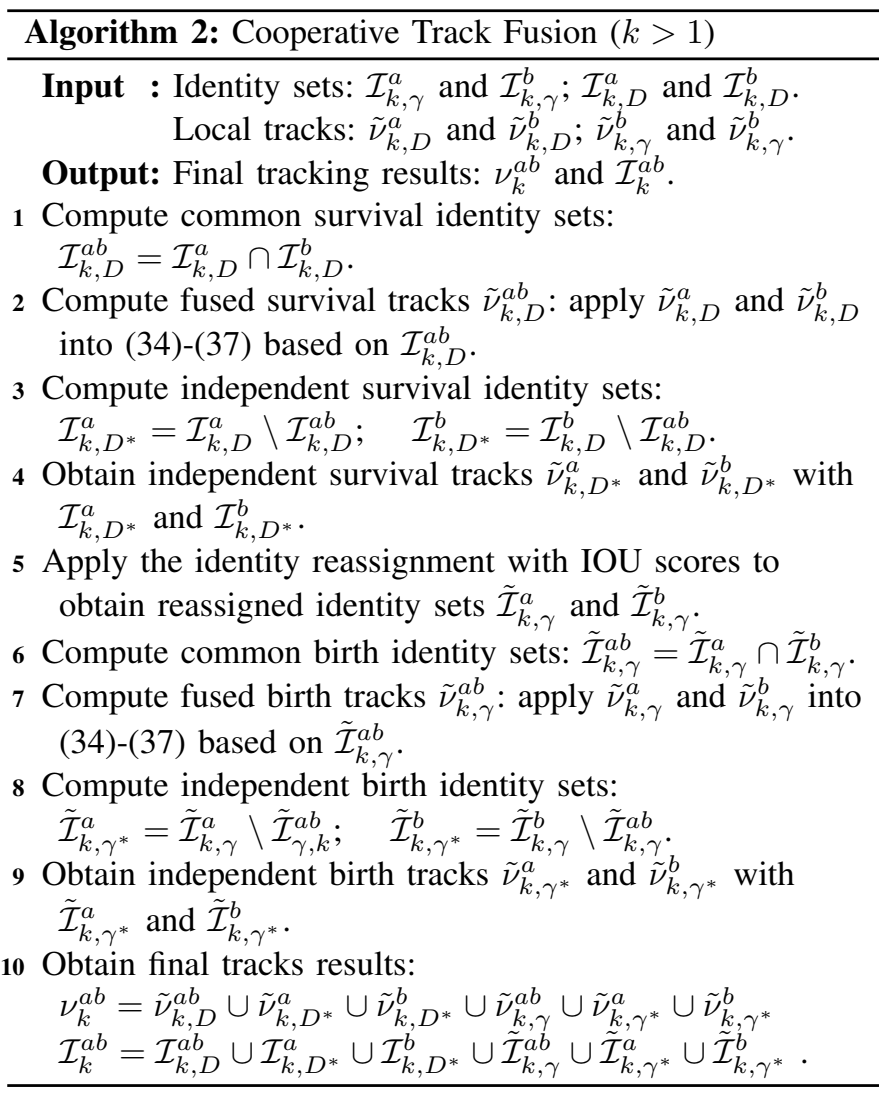

b) Birth Track Fusion: Different from survival track labelling system, a new-born target detected by each detector is labelled with two different local identities during the tracking process, which is due to the different detection orders in each detector. This is a issue of inconsistent identity assignment [27] which causes confusions in the fusion process. To remedy this, it is essential to reassign a same identity to matched birth tracks from both detectors. An identity reassignment mechanism is therefore developed before the fusion process, which is designed to calculate pairwise similarity score through intersection-over-union (IOU), i.e. $\operatorname{IOU}(a, b)=(\operatorname{Area}(a) \bigcap \operatorname{Area}(b)) /($ Area $(a) \bigcup \operatorname{Area}(b))$, between any two components from birth intensities of $\tilde{\nu}_{k, \gamma}^{a}(\mathbf{x})$ and $\tilde{\nu}_{k, \gamma}^{b}(\mathbf{x})[7]$. When the similarity score computed on any two birth components is greater than 0.5 , these two birth components will be reassigned with a same identity. Two components with the same reassigned identities from $\tilde{\nu}_{k, \gamma}^{a}(\mathbf{x})$ and $\tilde{\nu}_{k, \gamma}^{b}(\mathbf{x})$, respectively can be fused by the similar reasoning in 34)-37]. In addition, non-fused birth tracks are copied into the final tracking results.

2) Model Update: After the fusion process, it is necessary to update the appearance model for the newly achieved track- 
ing estimates in order to handle the appearance variations. To avoid introducing background noise in the model update, since the correlation filters are sensitive to false positives, our strategy is to only update the DCFs with the fused survival tracks $\tilde{\nu}_{k, D}^{a b}$ in the real zone. We specifically adopt the update mechanism in [47] for updating the DCF $\mathbf{c}_{k, d}^{(l)}$ on the $l$-th layer. Besides, each newly-detected track will be initialized with an appearance model using DCFs.

\section{B. Virtual Zone}

In this section, the virtual zone is designed to manage further validation and processing on the potentially missed tracks which are strictly excluded from the fusion process as shown in Fig. 4. The two major tasks of track reconfirmation and track termination are included in this zone.

1) Track Reconfirmation: Potentially missed tracks in the virtual zone are required for further reconfirmation by communicating the non-fused survival tracks from the real zone. Typically, we can reconfirm a track only if its identity can be found in the non-fused survival tracks from each detector domain. Then the reconfirmed track are removed from the virtual zone. However, tracks yet reconfirmed are considered as tentative tracks.

2) Track Termination: Tracks which are found from neither detectors are automatically moved into tentative tracks. Tentative tracks missing more than $T_{\text {miss }}$ frames are eliminated. Note that tentative tracks remaining in the virtual zone do not contribute to the final tracks, but they are propagated in the next time step.

\section{EXPERIMENTS}

In this section, we first briefly present the well-established datasets and evaluation metrics, then elaborate the detailed setting of our tracker implementation. Next, we conduct experiments on the validation sequences from the MOT16 benchmark to investigate the impact of the proposed fusion at different levels and the influence of different parameters on the overall tracking performance. Lastly, we evaluate the performance of the proposed method on the test set of MOT16 and MOT17 benchmarks.

\section{A. Datasets}

We conduct experiments on the MOTChallenge Benchmark datase ${ }^{1}$ which is the most commonly used for the quantification of multiple human tracking. This benchmark collects various challenging video sequences recorded by static or moving cameras, and under the complex scenes of illumination changes, varying viewpoints and weather conditions. The MOT16 Challenge [48], consists of 7 training and 7 testing fully annotated video sequences, as well as providing public object detections generated by [40] for fair comparisons. MOT17 Challenge [48] is built on the MOT16 Challenge with a new and more accurate ground truth. Each sequence is provided with 3 sets of public detections (DPM, FRCNN,

\footnotetext{
${ }^{1}$ https://motchallenge.net/
}

and SDP). The training video sequences with available ground truths are primarily utilized to process the performance analysis, while testing sequences are used to generate quantitative comparisons against existing state-of-the-art tracking methods.

\section{B. Evaluation Metrics}

We follow the widely-used evaluation metrics from the MOT benchmark to evaluate the proposed tracking method, including multiple object tracking accuracy (MOTA) [49], total number of false positives (FP), total number of false negatives (FN), total number of identity switches (IDS), mostly tracked (MT), mostly lost (ML), multiple object tracking precision (MOTP), total number of times a trajectory is fragmented (Frag), and the tracking speed in frames per second $(\mathrm{Hz})$.

\section{Implementation Details}

In this work, we use the state transition model $\mathbf{F}_{k}=$ $\left[\mathbf{I}_{2}, \Delta k \times \mathbf{I}_{2}, \mathbf{0}_{2} ; \mathbf{0}_{2}, \mathbf{I}_{2}, \mathbf{0}_{2} ; \mathbf{0}_{2}, \mathbf{0}_{2}, \mathbf{I}_{2}\right]$ with its process noise covariance $\mathbf{Q}_{k-1}=\operatorname{Diag}([25,25,16,16,4,4])$, where $\mathbf{I}_{2}$ and $\mathbf{0}_{2}$ are the $2 \times 2$ identity and zero matrices, and $\Delta k$ represents the time interval between frame $k$ and $k+1$. The observation model is $\mathbf{H}_{k}=\left[\mathbf{I}_{2}, \mathbf{0}_{2}, \mathbf{0}_{2} ; \mathbf{0}_{2}, \mathbf{0}_{2}, \mathbf{I}_{2}\right]$ and its observation noise covariance is $\mathbf{R}_{k}=\operatorname{Diag}([25,25])$ [2]. To implement the PHD filter, we empirically set $p_{M}=0.01, e=0.95$, and $\kappa=10^{-4}$ [5]. In order to reduce the effect of potentially noisy detections still existing in the measurement model, we follow the setting as in [50] to assign empirically a smaller value 0.001 for the birth weights. For the matching scheme, we set $\sigma_{c}=0.1$, the regularization parameter of 10$) \lambda=10^{-4}$, and $\sigma_{s}^{2}=30$ [16]. In addition, we experimentally determine the matching threshold $\varpi=10$ in Table III, so two coefficients $\alpha$ and $\beta$ in 14 are set to 0.2 and -2 , respectively. Likewise, the fusing weight parameter $\varphi$ is experimentally analyzed in Fig. 77. We employ the network in [51] for feature generation, where the outputs of the convolutional layers conv3-3 and conv4-3 are used as desired features. We multiply the extracted features with a cosine window to mitigate the boundary effect [16], [32]. We apply two settings for confidence scores, including $c_{t h}$ set to 0.1 for DPM detections, and set to $-\infty$ for FRCNN and SDP detections. The maximum missing frames $T_{\text {miss }}$ is set to 3 in this work, which is experimentally selected in Fig. 8.

\section{Performance Analysis}

In this section, we present the performance analysis to evaluate the effectiveness of our proposed method, including the ablation study of different proposed components as well as the effects of different parameter settings. For this purpose, we conduct experiments on MOT16-09 and MOT16-11 from the training set of MOT16 Challenge Benchmark [48], as the scene conditions and camera motions are distinct between these two validation sequences.

1) Ablation Study: To investigate the contribution of different components in our proposed method, we perform the ablation study in two aspects. Firstly, we compare the proposed method with single detector full-body (FB) or body parts 
(BP) individually processed by data-driven GM-PHD filter, and also the sequential update PHD fusion [14] with different detector orders, FB before $\mathrm{BP}$ (FB-BP) and $\mathrm{BP}$ before $\mathrm{FB}$ (BPFB). Next, we analyze the impact of different feature models in enhanced identity association including spatio-temporal information (ST) and discriminative correlation matching (A).

TABLE I

ABLATION STUDY OF OUR PROPOSED METHOD ON MOT16-09 SEQUENCE.

\begin{tabular}{|l|l|l|l|l|l|l|l|}
\hline Feature & Processer & $\begin{array}{l}\text { MOTA } \\
(\uparrow)\end{array}$ & $\begin{array}{l}\text { FP } \\
(\downarrow)\end{array}$ & $\begin{array}{l}\text { FN } \\
(\downarrow)\end{array}$ & $\begin{array}{l}\text { IDS } \\
(\downarrow)\end{array}$ & $\begin{array}{l}\text { MT } \\
(\uparrow)\end{array}$ & $\begin{array}{l}\text { ML } \\
(\downarrow)\end{array}$ \\
\hline \hline ST & FB & 29.4 & 1603 & 1981 & 128 & $32.0 \%$ & $16.0 \%$ \\
ST & BP & 33.1 & 1461 & 1943 & 112 & $16.0 \%$ & $8.0 \%$ \\
ST & FB-BP & 32.8 & 1839 & $\mathbf{1 5 7 3}$ & 122 & $\mathbf{3 6 . 0 \%}$ & $12.0 \%$ \\
ST & BP-FB & 34.9 & 1483 & 1814 & 125 & $24.0 \%$ & $8.0 \%$ \\
ST & Proposed & $\mathbf{3 9 . 9}$ & $\mathbf{1 1 1 2}$ & 1948 & $\mathbf{1 0 0}$ & $28.0 \%$ & $\mathbf{8 . 0 \%}$ \\
\hline A & FB & 34.6 & 1279 & 2036 & 124 & $\mathbf{2 8 . 0 \%}$ & $16.0 \%$ \\
A & BP & 39.4 & 1100 & 1983 & 101 & $12.0 \%$ & $\mathbf{8 . 0 \%}$ \\
A & FB-BP & 38.5 & 1208 & 1923 & 102 & $20.0 \%$ & $16.0 \%$ \\
A & BP-FB & 41.1 & 1137 & $\mathbf{1 8 4 7}$ & 111 & $24.0 \%$ & $12.0 \%$ \\
A & Proposed & $\mathbf{4 5 . 5}$ & $\mathbf{9 1 9}$ & 1850 & $\mathbf{9 8}$ & $24.0 \%$ & $12.0 \%$ \\
\hline ST+A & FB & 37.4 & 1136 & 2031 & 123 & $28.0 \%$ & $16.0 \%$ \\
ST+A & BP & 41.0 & 1012 & 1994 & 97 & $16.0 \%$ & $8.0 \%$ \\
ST+A & FB-BP & 40.8 & 1217 & 1798 & 95 & $24.0 \%$ & $12.0 \%$ \\
ST+A & BP-FB & 43.0 & 1108 & $\mathbf{1 7 7 5}$ & 110 & $20.0 \%$ & $12.0 \%$ \\
ST+A & Proposed & $\mathbf{4 7 . 5}$ & $\mathbf{8 2 7}$ & 1838 & $\mathbf{9 3}$ & $\mathbf{2 8 . 0 \%}$ & $\mathbf{8 . 0 \%}$ \\
\hline
\end{tabular}

TABLE II

AbLATION STUdy OF OUR PROPOSED METHOD ON MOT16-11 SEQUENCE.

\begin{tabular}{|l|l|l|l|l|l|l|l|}
\hline Feature & Processer & $\begin{array}{l}\text { MOTA } \\
(\uparrow)\end{array}$ & $\begin{array}{l}\text { FP } \\
(\downarrow)\end{array}$ & $\begin{array}{l}\text { FN } \\
(\downarrow)\end{array}$ & $\begin{array}{l}\text { IDS } \\
(\downarrow)\end{array}$ & $\begin{array}{l}\text { MT } \\
(\uparrow)\end{array}$ & $\begin{array}{l}\text { ML } \\
(\downarrow)\end{array}$ \\
\hline \hline ST & FB & 30.7 & 2379 & 3871 & 111 & $21.7 \%$ & $42.0 \%$ \\
ST & BP & 36.1 & 2155 & $\mathbf{3 6 0 7}$ & 102 & $23.1 \%$ & $34.7 \%$ \\
ST & FB-BP & 35.2 & 2147 & 3675 & 122 & $24.6 \%$ & $40.5 \%$ \\
ST & BP-FB & 37.9 & 1758 & 3845 & 95 & $20.2 \%$ & $43.4 \%$ \\
ST & Proposed & $\mathbf{4 1 . 9}$ & $\mathbf{1 6 2 5}$ & 3622 & $\mathbf{8 6}$ & $\mathbf{2 6 . 1 \%}$ & $\mathbf{3 1 . 9 \%}$ \\
\hline A & FB & 36.1 & 1808 & 3953 & 100 & $20.3 \%$ & $42.0 \%$ \\
A & BP & 41.2 & 1637 & 3662 & 94 & $20.3 \%$ & $\mathbf{3 4 . 7 \%}$ \\
A & FB-BP & 40.5 & 1775 & 3574 & 108 & $16.0 \%$ & $44.9 \%$ \\
A & BP-FB & 43.0 & 1633 & 3507 & 90 & $20.2 \%$ & $43.4 \%$ \\
A & Proposed & $\mathbf{4 8 . 1}$ & $\mathbf{1 3 5 9}$ & $\mathbf{3 3 2 4}$ & $\mathbf{8 0}$ & $\mathbf{2 1 . 7 \%}$ & $36.2 \%$ \\
\hline ST+A & FB & 38.2 & 1776 & 3793 & 97 & $18.9 \%$ & $43.4 \%$ \\
ST+A & BP & 42.3 & 1630 & 3576 & 91 & $23.1 \%$ & $\mathbf{3 4 . 7 \%}$ \\
ST+A & FB-BP & 41.1 & 1713 & 3596 & 98 & $\mathbf{2 4 . 6 \%}$ & $40.5 \%$ \\
ST+A & BP-FB & 43.7 & 1573 & 3496 & 91 & $21.7 \%$ & $39.1 \%$ \\
ST+A & Proposed & $\mathbf{4 9 . 7}$ & $\mathbf{1 2 6 6}$ & $\mathbf{3 2 7 8}$ & $\mathbf{7 4}$ & $24.6 \%$ & $37.7 \%$ \\
\hline
\end{tabular}

Tables II and II report the detailed evaluations on the validation sequences. In general, we can see from the results above, the full tracking model achieves noticeably improved performance on almost all presented evaluation metrics. On the one hand, the proposed cooperative track fusion shows the advantage over the sequential fusion approaches (FB-BP \& BP-FB) and single detectors (FB \& BP), as it improves MOTA and reduces the number of FNs regardless of different feature models. This is because the proposed fusion algorithm exploits well the merits of both human detectors, thereby enabling the tracker to recover the missed detections and provide more reliably consistent tracks. Another finding on these results is that the proposed discriminative correlation matching (A) mainly contributes to reducing the number of FPs, and ID switches. This may be explained by the fact that the proposed appearance model could help the tracking system to establish better mappings between the detections and real targets.
MOT16-09: Static camera

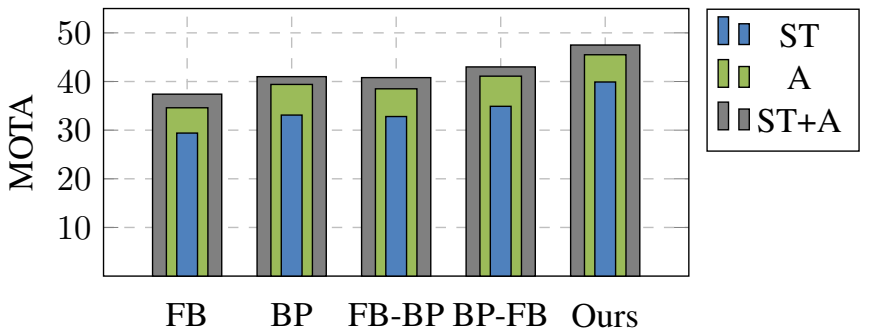

(a)

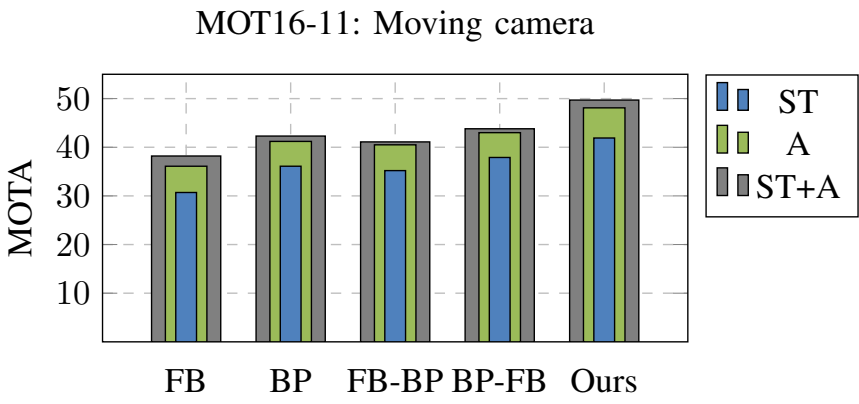

(b)

Fig. 6. MOTA performance comparison of our proposed method on the validation sequences.

Fig. 6 intuitively reveals the advantage of our feature-level fusion. The combined model achieves the best MOTA performance regardless of different fusion solutions, suggesting that fusing features can improve ambiguities which occur in either motion dynamics or visual content. It is shown that the proposed appearance model contributes most to improve the tracking performance. Moreover, spatio-temporal information helps increase the overall accuracy, especially to facilitate localization of the targets with similar appearances. Overall, ablation study results above verify the proposed multi-level fusion is helpful to address target ambiguities, and provide redundancy in each detector domain.

2) Analysis of Parameters: We also conduct experiments on the validation sequences to analyze the influence of different critical parameters on the tracking performance. We first test four different values of matching threshold $\varpi$ which controls the gate to accept the matched pairs, as illustrated in Table III Metrics of MOTA, FP, and FN are employed to investigate the relative change on the performance. For the MOT16-09 sequence, when the value of $\varpi$ is altered from 6 to 18 , the number of false positives is largely decreased, whereas the MOTA score is just slightly improved. This is because the higher matching threshold ignores some matched pairs in ambiguous cases. Similar results are also found on the MOT16-11 sequence.

In addition, we analyze the fusing parameter $\varphi$ which determines the relative fusion weight of each detector with 5 different settings, and the results of different $\varphi$ are as shown in Fig. 7. Since both detectors have different detecting abilities, the tracking performance is slightly sensitive to the fusing parameter. As we can see from the extreme cases, the full body detector can provide better precision MOTP 
TABLE III

COMPARISON OF THE TRACKING RESULTS WITH DIFFERENT MATCHING THRESHOLDS OF APPEARANCE MODEL ON THE VALIDATION SEQUENCES

\begin{tabular}{|c|c|c|c|c|}
\hline \multicolumn{5}{|c|}{ МOT16-09 } \\
\hline$\varpi$ & 6 & 10 & 14 & 18 \\
\hline MOTA ( $\uparrow)$ & 44.3 & 47.5 & 46.5 & 45.9 \\
\hline FP $(\downarrow)$ & 1149 & 827 & 754 & 676 \\
\hline FN $(\downarrow)$ & 1667 & 1838 & 1958 & 2083 \\
\hline \multicolumn{5}{|c|}{ MOT16-11 } \\
\hline$\varpi$ & 6 & 10 & 14 & 18 \\
\hline MOTA $(\uparrow)$ & 46.0 & 49.7 & 48.5 & 49.1 \\
\hline FP $(\downarrow)$ & 1614 & 1266 & 1146 & 1019 \\
\hline FN $(\downarrow)$ & 3220 & 3278 & 3493 & 3582 \\
\hline
\end{tabular}

but less accuracy MOTA, while the body-parts detector has the opposite impact on the performance. To this end, we experimentally determine an appropriate value for $\varphi$ to manage the trade off between the MOTA and MOTP. To study the impact of the parameter $T_{m i s s}$ which controls the number of consecutive missing frames to terminate tentative tracks, we ran a set of pilot tests $T_{\text {miss }}=\{1,3,5,7,9\}$ on the validation sequences. The frame rate used in both sequences is 30 , which is obtained from the MOT16 Challenge [48]. The results in Fig. 8 show that the best setting for the maximum missing frames is $T_{\text {miss }}=3$. The above analysis demonstrates that the tracking performance of our proposed method is slightly sensitive to the parameter changes in the reasonable range. Note that we use parameters with the highest performance and keep them unchanged throughout the experiments.

MOT16-09

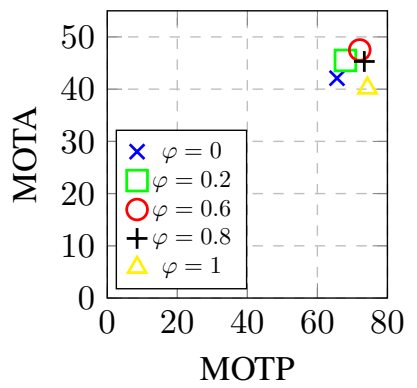

(a)

Fig. 7. Comparisons of MOTA performance with different fusing parameters $\varphi$ on the validation sequences. Results closer to the upper right corner perform better(better viewed in color version).

\section{E. Benchmark Evaluations}

We evaluate the proposed tracking system on the test set of the MOT16 and MOT17 Challenge Benchmarks [48]. Quantitative results compared with recent state-of-the-art trackers published on the leaderboard are shown in Tables IV and $\mathrm{V}$. These include online trackers: AMIR [9], DCCRF16 [13], CDA_DDALv2 [52], Deep-align [1], EAMTTPub [15], GM_PHD_N1T [17], GMPHD_HDA [31], MOTDT [11], PHD_GSDL [5] and GMPHD_KCF [20], GM_PHD [21], and also offline trackers: INTERA_MOT [12], FWT [8], MCjoint

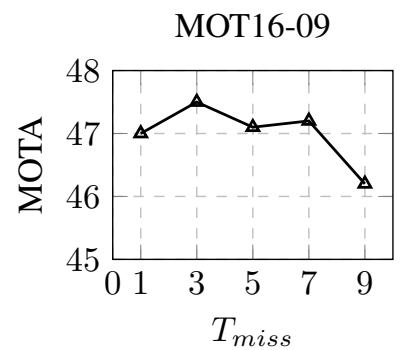

(a)

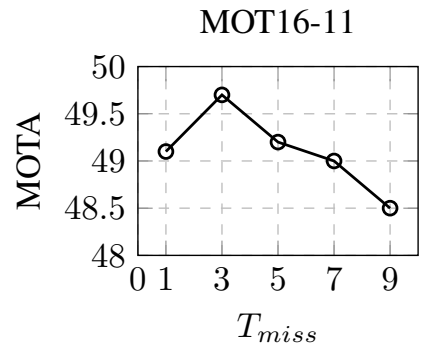

(b)
Fig. 8. Comparisons of MOTA performance with different values of parameter $T_{\text {miss }}$ on the validation sequences.

[53], MHT_DAM [54], EDMT [55], QuadMOT16 [56], and MHT_bLSTM [30]. Evaluation measures with $(\uparrow)$ or $(\downarrow)$ respectively denote that higher is better, or lower is better. We maximize the MOTA score which is regarded as the most important measure for the overall ranking. We also show the qualitative tracking results on the MOT17 dataset in Fig. 9

As we can see from Table IV, the proposed method (MTDF) reports the state-of-the-art MOTA and the second best MT compared with online methods, which indicates our method is capable to provide more reliably consistent tracks. Likewise, MTDF achieves the lowest ML and FN, even including offline methods, demonstrating that our method has the advantage to recover missed targets by fusing parts to a cohesive whole. Note that offline methods using future frame information usually achieve more promising performance than online methods. Our improved tracking performance continues with the evaluation on the MOT17 Benchmark, as shown in Table V] MTDF17 achieves the second best performance in MOTA, best MT among published online methods, and is on a par with state-of-the-art offline methods. Furthermore, our method records the best ML and FN scores among all listed trackers here. As a trade off, the proposed method produces more FPs. According to [49], false positives can be defined as tracking hypotheses which do not have correspondence with real objects. For instance, objects which are not humans tracked as humans or there are no desired objects in the tracked bounding boxes.

\section{F. Discussions with other MOT methods}

In this section, we explicitly discuss benchmark performance between the proposed approach and other MOT methods. Compared with a similar approach [8], we achieve better ML and FN, while their fusion approach formulated by a quadratic program performs better in MT and FP due to exploiting the long-term latency. It is worth noting that the proposed method is outperformed by MOTDT [11], which specifically achieves more promising performance in MOTA, FP, ID Sw, and Frag. The effectiveness of this tracker can be attributed to two advantages, one is that a fully CNN based candidate selection is well designed to remove the false positives in the early stage, thereby gaining more reliable detections for the data association. The other is triplet-based person re-identification improves the target appearance model 
TABLE IV

QUANTITATIVE COMPARISON BETWEEN OUR APPROACH (MTDF) AND STATE-OF-THE-ART APPROACHES ON THE MOT16 BENCHMARK. THE BEST RESULTS OF ONLINE OR OFFLINE APPROACHES ARE SHOWN IN BOLD RESPECTIVELY. (LAS SUBMITTED ON MAY 15, 2018)

\begin{tabular}{|c|c|c|c|c|c|c|c|c|c|c|}
\hline Method & Mode & MOTA $(\uparrow)$ & MOTP $(\uparrow)$ & MT ( $(\uparrow)$ & ML $(\downarrow)$ & FP $(\downarrow)$ & FN $(\downarrow)$ & ID Sw ( $(\downarrow)$ & Frag $(\downarrow)$ & $\mathrm{Hz}(\uparrow)$ \\
\hline MOTDT $\sqrt{11}$ & Online & 47.6 & 74.8 & $15.2 \%$ & $38.3 \%$ & 9,253 & 85,431 & 792 & 1,858 & 20.6 \\
\hline AMIR $[9]$ & Online & 47.2 & 75.8 & $14.0 \%$ & $41.6 \%$ & 2,681 & 92,856 & 774 & 1,675 & 1.0 \\
\hline MTDF & Online & 45.7 & 72.6 & $14.1 \%$ & $36.4 \%$ & 12,018 & 84,970 & 1,987 & 3,377 & 1.5 \\
\hline DCCRF16 [13] & Online & 44.8 & 75.6 & $14.1 \%$ & $42.3 \%$ & 5,613 & 94,133 & 968 & 1,378 & 0.1 \\
\hline CDA_DDALv2 $52 \mid$ & Online & 43.9 & 74.7 & $10.7 \%$ & $44.4 \%$ & 6,450 & 95,175 & 676 & 1,795 & 0.5 \\
\hline Deep-align $|1|$ & Online & 40.8 & 74.4 & $13.7 \%$ & $38.3 \%$ & 15143 & 91792 & 1051 & 2210 & 6.5 \\
\hline EAMTT_pub [15] & Online & 38.8 & 75.1 & $7.9 \%$ & $49.1 \%$ & 8,114 & 102,452 & 965 & 1,657 & 11.8 \\
\hline GM_PHD_N1T [17] & Online & 33.3 & 76.8 & $5.5 \%$ & $56.0 \%$ & 1,750 & 116,452 & 3,499 & 3,594 & 9.9 \\
\hline GMPHD_HDA $\mid 31]$ & Online & 30.5 & 75.4 & $4.6 \%$ & $59.7 \%$ & 5,169 & 120,970 & 539 & 731 & 13.6 \\
\hline FWT [8] & Offline & 47.8 & 75.5 & $19.1 \%$ & $38.2 \%$ & 8,886 & 85,487 & 852 & 1,534 & 0.6 \\
\hline MCjoint [53] & Offline & 47.1 & 76.3 & $20.4 \%$ & $46.9 \%$ & 6,703 & 89,368 & 370 & 598 & 0.6 \\
\hline MHT_DAM |54| & Offline & 45.8 & 76.3 & $16.2 \%$ & $43.2 \%$ & 6,412 & 91,758 & 590 & 781 & 0.8 \\
\hline INTERA_MOT |1 & Offline & 45.4 & 74.4 & $18.1 \%$ & $38.7 \%$ & 13,407 & 85,547 & 600 & 930 & 4.3 \\
\hline EDMT [55] & Offline & 45.3 & 75.9 & $17.0 \%$ & $39.9 \%$ & 11,122 & 87,890 & 639 & 946 & 1.8 \\
\hline QuadMOTi6 [56] & Offline & 44.1 & 76.4 & $14.6 \%$ & $44.9 \%$ & 6,388 & 94,775 & 745 & 1,096 & 1.8 \\
\hline
\end{tabular}

TABLE V

QUANTITATIVE COMPARISON BETWEEN OUR APPROACH (MTDF17) AND STATE-OF-THE-ART APPROACHES ON THE MOT17 BENCHMARK. THE BEST RESULTS OF ONLINE OR OFFLINE APPROACHES ARE SHOWN IN BOLD RESPECTIVELY. (LAS SUBMITTED ON MAY 23, 2018)

\begin{tabular}{|c|c|c|c|c|c|c|c|c|c|c|}
\hline Method & Mode & MOTA $(\uparrow)$ & MOTP $(\uparrow)$ & MT $(\uparrow)$ & ML $(\downarrow)$ & FP $(\downarrow)$ & FN $(\downarrow)$ & ID Sw $(\downarrow)$ & Frag $(\downarrow)$ & Hz $(\uparrow)$ \\
\hline \hline MOTDT17 [11] & Online & $\mathbf{5 0 . 9}$ & 76.6 & $17.5 \%$ & $35.7 \%$ & 24,069 & 250,768 & $\mathbf{2 , 4 7 4}$ & 5,317 & 18.3 \\
\hline MTDF17 & Online & 49.6 & 75.5 & $\mathbf{1 8 . 9 \%}$ & $\mathbf{3 3 . 1 \%}$ & 37,124 & $\mathbf{2 4 1 , 7 6 8}$ & 5,567 & 9,260 & 1.2 \\
\hline PHD_GSDL17 $[5]$ & Online & 48.0 & $\mathbf{7 7 . 2}$ & $17.1 \%$ & $35.6 \%$ & $\mathbf{2 3 , 1 9 9}$ & 265,954 & 3,998 & 8,886 & 6.7 \\
EAMTT [15] & Online & 42.6 & 76.0 & $12.7 \%$ & $42.7 \%$ & 30,711 & 288,474 & 4,488 & $\mathbf{5 , 7 2 0}$ & 1.4 \\
GMPHD_KCF[20] & Online & 39.6 & 74.5 & $8.8 \%$ & $43.3 \%$ & 50,903 & 284,228 & 5,811 & 7,414 & 3.3 \\
GM_PHD [21] & Online & 36.4 & 74.5 & $4.1 \%$ & $57.3 \%$ & 23,723 & 330,767 & 4,607 & 11,317 & $\mathbf{3 8 . 4}$ \\
\hline \hline FWT[8] & Offline & $\mathbf{5 1 . 3}$ & 75.9 & $21.4 \%$ & $\mathbf{3 5 . 2 \%}$ & 24,101 & 247,921 & 2648 & 4279 & 0.2 \\
jCC [53] & Offline & 51.2 & 75.9 & $20.9 \%$ & $37.0 \%$ & 25,937 & 247,822 & $\mathbf{1 , 8 0 2}$ & 2,984 & 1.8 \\
MHT_DAM [54] & Offline & 50.7 & $\mathbf{7 7 . 5}$ & $20.8 \%$ & $36.9 \%$ & $\mathbf{2 2 , 8 7 5}$ & 252,889 & 2,314 & $\mathbf{2 , 8 6 5}$ & 0.9 \\
EDMT17 [55] & Offline & 50.0 & 77.3 & $\mathbf{2 1 . 6 \%}$ & $36.3 \%$ & 32,279 & $\mathbf{2 4 7 , 2 9 7}$ & 2,264 & 3,260 & 0.6 \\
MHT_bLSTM[30] & Offline & 47.5 & 77.5 & $18.2 \%$ & $41.7 \%$ & 25,981 & 268,042 & 2,069 & 3,124 & $\mathbf{1 . 9}$ \\
\hline
\end{tabular}

with better discriminativity, so as to reduce the number of ID switches and fragments.

Moreover, we specifically compare the proposed method against other state-of-the-art RFS based methods published on the leaderboard, including EAMTTPub [15], GM_PHD_N1T [17], GMPHD_HDA [31], PHD_GSDL17 [5], GM_PHD [21], and GMPHD_KCF [20]. Overall, our method achieves best tracking performance among all RFS based methods in both benchmarks. Comparing to our previous work PHD_GSDL17 [5] in Table V] the proposed approach here effectively reduces a large amount of FN, and improves MOTA by $1.6 \%$, MT by $1.8 \%$ and ML by $2.5 \%$. The improvement over EAMTT [15], which also performs early association but only using spatial constraints, verifies the benefits of integrating the targetspecific appearance model within our approach, particularly in establishing much more reliable and stable tracks. Better yet, the proposed method outperforms single-level fusion based approaches in [21] and [20] with large margins on the MOT17 dataset, demonstrating that the proposed multi-level tracking fusion can increase the robustness and reliability for multiple human tracking task. Evaluations above imply our multilevel fusion approach can considerably strengthen RFS based multiple human tracking.

To analyze the advantages of the currently proposed approach over methods given in previous conference papers [7] and [16], we explicitly evaluated their tracking performance on the MOT16 benchmark. The results in Table VI demonstrate the proposed approach achieves the best scores in terms of MOTA, MT, ML, and FN. The improved performance can be attributed to the major contribution which is the proposed fusion center. It enhances the overall fusion process and contributes to producing lower scores in FN, ML and better MOTA performance. Other improvements ensue from the proposed multi-level cooperative fusion method integrating well the merits from previous works at different stages. By exploiting the complementary benefits of using the two human detectors in [7], more reliable tracks are provided, which yields improved MT score. The work in [16] which improves the identity association helps the tracker to produce better performance in ID Sw and Frag. As a suggestion for future work, FP in the proposed fusion approach can be further improved by exploiting the long-term latency of target trajectories.

\section{G. Runtime Analysis}

In this work, all the experiments were conducted on a Laptop with an Intel i7 $3.5 \mathrm{GHz} \mathrm{CPU}$, with $32 \mathrm{~GB}$ of memory and a GeForce GTX 1060 without parallel speeding. We summarize the runtime comparisons with other published methods presented in the MOT Benchmark in Tables IV] and $\mathrm{V}$. The proposed method returns a longer runtime performance compared with the existing RFS based methods, such as EAMTTPub [15], GMPHD_KCF [20] and GM_PHD [21]. 

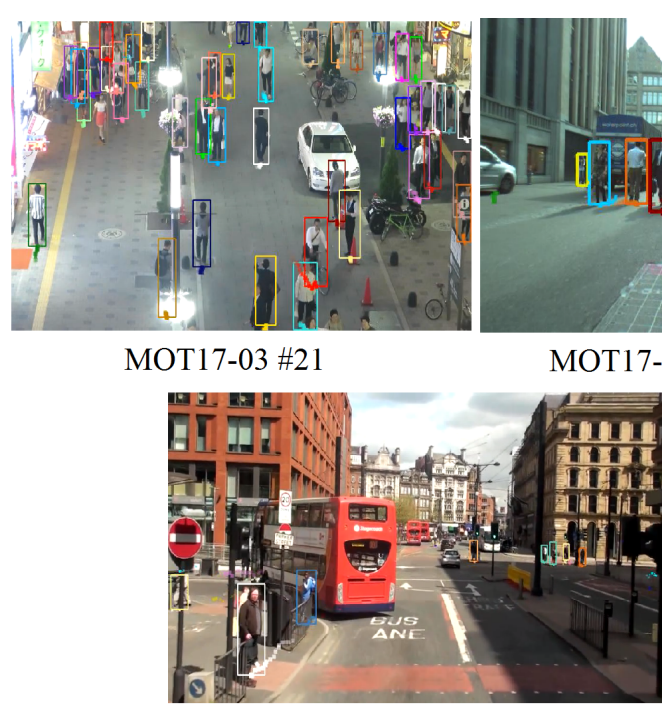

MOT17-14 \#143

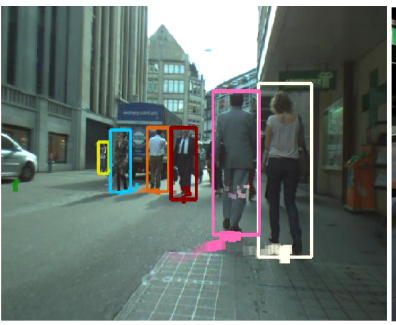

MOT17-06 \#611

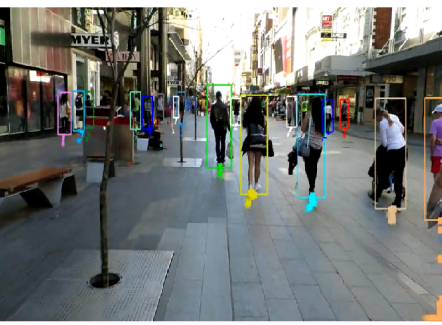

MOT17-07 \#67

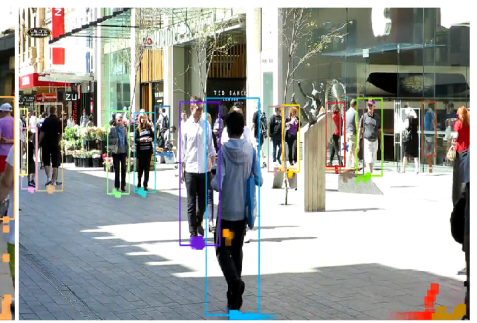

MOT17-08 \#95

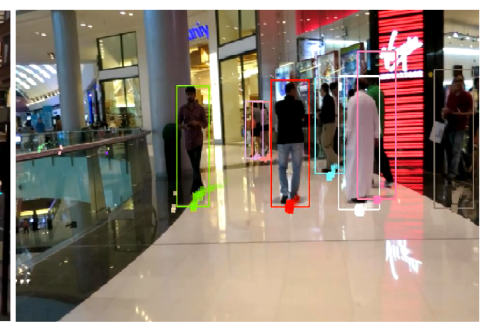

MOT17-12 \#159

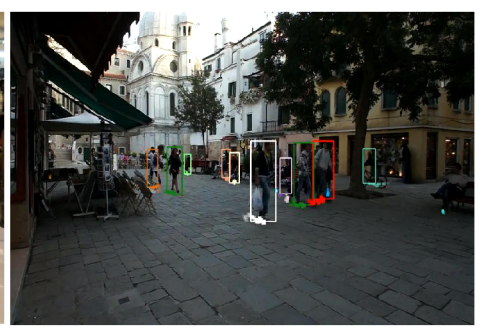

MOT17-01 \#255

Fig. 9. Visual tracking results of our proposed tracking system on the test set of MOT17 dataset. Different colors of the bounding boxes represent identities.

TABLE VI

QUANTITATIVE COMPARISON BETWEEN THE PROPOSED APPROACH (MTDF) AND PREVIOUSLY PUBLISHED VERSIONS ON THE MOT16 BENCHMARK.

\begin{tabular}{|c|c|c|c|c|c|c|c|c|}
\hline Method & MOTA $(\uparrow)$ & MT $(\uparrow)$ & ML $(\downarrow)$ & FP $(\downarrow)$ & FN $(\downarrow)$ & ID Sw $(\downarrow)$ & Frag $(\downarrow)$ & Hz $(\uparrow)$ \\
\hline MTDF & $\mathbf{4 5 . 7}$ & $\mathbf{1 4 . 1 \%}$ & $\mathbf{3 6 . 4 \%}$ & 12,018 & $\mathbf{8 4 , 9 7 0}$ & 1,987 & 3,377 & 1.5 \\
\hline$|\overline{7}|$ & 39.3 & $12.5 \%$ & $40.8 \%$ & 12,430 & 93,394 & 4,934 & 5,886 & $\mathbf{9 . 7}$ \\
\hline$|\overline{16}|$ & 37.7 & $9.2 \%$ & $46.5 \%$ & $\mathbf{6 , 5 1 5}$ & 105,389 & $\mathbf{1 6 0 8}$ & $\mathbf{3 , 3 7 2}$ & 2.2 \\
\hline
\end{tabular}

In [15], target appearance models which usually consume the most computations were not applied in the association. This increases the tracking speed but generates less promising tracking results. In [20] and [21], single-level based fusion trackers using hand-crafted features for appearance modelling were used, in a sense, which can reduce the computational complexity. To further analyse the computation cost of our multi-level fusion system, we provide runtime comparisons between the journal version and previous conference papers in Table VI. We discover that the most consuming component is within the enhanced identity association, particularly in the use of the appearance term. This can be comprehended from the runtime of [16], computing the target appearance models and their update in a many to many scenario clearly slows down the running speed, especially when the environment becomes much congested. However, the collaborative detector fusion approach [7] can run faster by disabling the expensive appearance matching.

\section{H. Failure Cases}

In Fig. 10, we present selected tracking failure cases of our proposed method. In Fig. 10 (a), it is clear to see that when a target is occluded for a longer time, it will be labelled with a new identity after occlusion. For instance, the woman on the left is initially labelled with a purple bounding box. When she reappears after occlusion, she is initialized as a new born target with a newly assigned green box. This can be further addressed by maintaining the long-term memory of appearance models for potentially disappeared targets. In addition, it is not difficult to see in Fig. 10 (b), two people switch their IDs on the right. A man in black shirt is initially labelled with a green bounding box, but his identity (green box) is shifted to the man in white shirt in the next frames. This motivates us to incorporate better deep features in the correlation matching, such as in [57] and [58], in order to better discriminate targets within the occlusion region. Alternatively, occlusion-aware detection [59] can be used in the future work to redetect the targets after long-term occlusion.

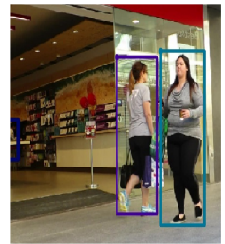

(a) MOT16-09

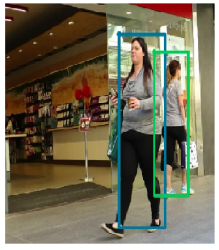

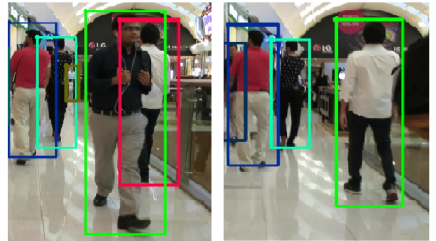

(b) MOT16-11
Fig. 10. Selected tracking failure cases of our proposed method.

\section{CONCLUSION}

In this paper, we presented a novel online multiple human tracking method using multi-level cooperative fusion with data-driven GM-PHD filters. For the feature level fusion, we firstly exploited discriminative correlation filters with multilayer convolutional features as target-specific classifiers which discriminate targets as either inter-class or intra-class. This appearance model is fused with spatio-temporal information to effectively mitigate the association ambiguities when targets are in close proximity. For the decision level fusion, 
we developed a new fusion strategy with real and virtual zones to perform the fusions on the survival and birth tracks, which alleviates the issue of missed detections in the GCI fusion rule thereby maximizing the complementary benefits using both detectors. As a whole, such a multi-level fusion approach can simultaneously acquire more reliable tracks and recover missed targets under various challenging scenarios. Performance analysis and evaluations on the MOTChallenge were shown to demonstrate the effectiveness of the proposed approach as well as the improved performance particularly in FN and ML. Future work will explore long-term target dependencies to further tackle the long-term occlusions.

\section{ACKNOWLEDGMENT}

The authors would like to thank Professor Ling Shao, Associate Editor and the anonymous reviewers for their valuable input to improving the paper.

\section{REFERENCES}

[1] Q. Zhou, B. Zhong, Y. Zhang, J. Li, and Y. Fu, "Deep alignment network based multi-person tracking with occlusion and motion reasoning," IEEE Transactions on Multimedia, pp. 1-13, 2018.

[2] P. Feng, W. Wang, S. Dlay, S. M. Naqvi, and J. Chambers, "Social Force Model-Based MCMC-OCSVM Particle PHD Filter for Multiple Human Tracking," IEEE Transactions on Multimedia, vol. 19, no. 4, pp. 725-739, 2017.

[3] Q. Liu, W. Wang, T. de Campos, P. J. B. Jackson, and A. Hilton, "Multiple Speaker Tracking in Spatial Audio via PHD Filtering and Depth-Audio Fusion," IEEE Transactions on Multimedia, pp. 1-1, 2017.

[4] A. Ur-Rehman, S. M. Naqvi, L. Mihaylova, and J. A. Chambers, "MultiTarget Tracking and Occlusion Handling with Learned Variational Bayesian Clusters and a Social Force Model," IEEE Transactions on Signal Processing, vol. 64, no. 5, pp. 1320-1335, 2016.

[5] Z. Fu, P. Feng, F. Angelini, J. Chambers, and S. M. Naqvi, "Particle PHD Filter based Multiple Human Tracking using Online Group-Structured Dictionary Learning," IEEE Access, vol. 6, pp. 14 764-14778, 2018.

[6] O. Khalid, J. C. SanMiguel, and A. Cavallaro, "Multi-Tracker Partition Fusion," IEEE Transactions on Circuits and Systems for Video Technology, vol. 27, no. 7, pp. 1527-1539, 2017.

[7] Z. Fu, S. M. Naqvi, and J. A. Chambers, "Collaborative detector fusion of data-driven PHD filter for online multiple human tracking," in International Conference on Information Fusion (FUSION), 2018, pp. 1976-1981.

[8] R. Henschel, L. Leal-Taix, D. Cremers, and B. Rosenhahn, "Fusion of Head and Full-Body Detectors for Multi-Object Tracking," arXiv:1705.08314 [cs.CV], pp. 1-10, 2017.

[9] A. Sadeghian, A. Alahi, and S. Savarese, "Tracking the Untrackable: Learning to Track Multiple Cues with Long-Term Dependencies," in IEEE International Conference on Computer Vision (ICCV), 2017, pp. 300-311.

[10] S. Tang, B. Andres, M. Andriluka, and B. Schiele, "Multi-person Tracking by Multicut and Deep Matching," in European Conference on Computer Vision Workshops (ECCVW), 2016.

[11] L. Chen, H. Ai, Z. Zhuang, and C. Shang, "Real-time multiple people tracking with deeply learned candidate selection and person reidentification," in IEEE International Conference on Multimedia and Expo (ICME), 2018.

[12] L. Lan, X. Wang, S. Zhang, D. Tao, W. Gao, and T. S. Huang, "Interacting Tracklets for Multi-Object Tracking," IEEE Transactions on Image Processing, vol. 27, no. 9, pp. 4585-4597, 2018.

[13] H. Zhou, W. Ouyang, J. Cheng, X. Wang, and H. Li, "Deep Continuous Conditional Random Fields with Asymmetric Inter-object Constraints for Online Multi-object Tracking," IEEE Transactions on Circuits and Systems for Video Technology, pp. 1-1, 2018.

[14] R. P. S. Mahler, "Multitarget Bayes Filtering via First-Order Multitarget Moments," IEEE Transactions on Aerospace and Electronic Systems, vol. 39, no. 4, pp. 1152-1178, 2003.

[15] R. Sanchez-Matilla, F. Poiesi, and A. Cavallaro, "Online multi-target tracking with strong and weak detections," in European Conference on Computer Vision Workshops (ECCVW), 2016, pp. 84-99.
[16] Z. Fu, F. Angelini, S. M. Naqvi, and J. A. Chambers, "GM-PHD Filter Based Online Multiple Human Tracking Using Deep Discriminative Correlation Matching," in IEEE International Conference on Acoustics, Speech and Signal Processing (ICASSP), 2018, pp. 4299-4303.

[17] N. L. Baisa and A. Wallace, "Development of a N-type GM-PHD Filter for Multiple Target, Multiple Type Visual Tracking," arXiv:1706.00672 [cs.CV], pp. 1-17, 2017.

[18] R. Gravina, P. Alinia, H. Ghasemzadeh, and G. Fortino, "Multi-sensor fusion in body sensor networks: State-of-the-art and research challenges," Information Fusion, vol. 35, pp. 68 - 80, 2017.

[19] A. J. Ma, P. C. Yuen, and S. Saria, "Deformable Distributed Multiple Detector Fusion for Multi-Person Tracking," arXiv:1512.05990 [cs.CV], pp. 1-9, 2015.

[20] T. Kutschbach, E. Bochinski, V. Eiselein, and T. Sikora, "Sequential Sensor Fusion Combining Probability Hypothesis Density and Kernelized Correlation Filters for Multi-Object Tracking in video data," in IEEE International Workshop on Traffic and Street Surveillance for Safety and Security (AVSS), 2017, pp. 1-5.

[21] V. Eiselein, D. Arp, M. Ptzold, and T. Sikora, "Real-Time Multi-human Tracking Using a Probability Hypothesis Density Filter and Multiple Detectors," in IEEE International Conference on Advanced Video and Signal-Based Surveillance, 2012, pp. 325-330.

[22] M. Uney, D. E. Clark, and S. J. Julier, "Distributed Fusion of PHD Filters Via Exponential Mixture Densities," IEEE Journal of Selected Topics in Signal Processing, vol. 7, no. 3, pp. 521-531, 2013.

[23] G. Battistelli, L. Chisci, C. Fantacci, A. Farina, and A. Graziano, "Consensus CPHD Filter for Distributed Multitarget Tracking," IEEE Journal of Selected Topics in Signal Processing, vol. 7, no. 3, pp. 508520, 2013.

[24] T. A. Biresaw, A. Cavallaro, and C. S. Regazzoni, "Tracker-Level Fusion for Robust Bayesian Visual Tracking," IEEE Transactions on Circuits and Systems for Video Technology, vol. 25, no. 5, pp. 776-789, 2015.

[25] R. P. S. Mahler, "Optimal/robust distributed data fusion: a unified approach," Proc. SPIE Signal Processing, Sensor Fusion, and Target Recognition IX, vol. 4052, pp. 128-138, 2000.

[26] D. Clark, S. Julier, R. P. S. Mahler, and B. Ristic, "Robust multi-object sensor fusion with unknown correlations," in Sensor Signal Processing for Defence Conference (SSPD), 2010, pp. 1-5.

[27] B. Wang, W. Yi, S. Li, M. R. Morelande, L. Kong, and X. Yang, "Distributed Multi-target Tracking via Generalized Multi-Bernoulli Random Finite Sets," in International Conference on Information Fusion (FUSION), 2015, pp. 253-261.

[28] L. Chen, H. Ai, C. Shang, Z. Zhuang, and B. Bai, "Online multi-object tracking with convolutional neural networks," in IEEE International Conference on Image Processing (ICIP), 2017, pp. 645-649.

[29] J. Shen, Z. Liang, J. Liu, H. Sun, L. Shao, and D. Tao, "Multiobject tracking by submodular optimization," IEEE Transactions on Cybernetics, pp. 1-12, 2018.

[30] C. Kim, F. Li, and J. M. Rehg, "Multi-object tracking with neural gating using bilinear LSTM," in European Conference on Computer Vision (ECCV), 2018.

[31] Y. Song and M. Jeon, "Online multiple object tracking with the hierarchically adopted GM-PHD filter using motion and appearance," in IEEE International Conference on Consumer Electronics-Asia (ICCE-Asia), 2016, pp. 1-4.

[32] C. Ma, J. B. Huang, X. Yang, and M. H. Yang, "Hierarchical Convolutional Features for Visual Tracking," in IEEE International Conference on Computer Vision (ICCV), 2015, pp. 3074-3082.

[33] Y. Yang and G. A. Bilodeau, "Multiple object tracking with kernelized correlation filters in urban mixed traffic," in 14th Conference on Computer and Robot Vision (CRV), 2017, pp. 209-216.

[34] H. Wu and W. Li, "Robust online multi-object tracking based on kcf trackers and reassignment," in IEEE Global Conference on Signal and Information Processing (GlobalSIP), 2016, pp. 124-128.

[35] S. H. Park, K. Lee, and K. J. Yoon, "Robust online multiple object tracking based on the confidence-based relative motion network and correlation filter," in IEEE International Conference on Image Processing (ICIP), 2016, pp. 3484-3488.

[36] K. Panta, D. E. Clark, and B. N. Vo, "Data Association and Track Management for the Gaussian Mixture Probability Hypothesis Density Filter," IEEE Transactions on Aerospace and Electronic Systems, vol. 45, no. 3, pp. 1003-1016, 2009.

[37] X. Zhou, H. Yu, H. Liu, and Y. Li, "Tracking Multiple Video Targets with an Improved GM-PHD Tracker," Sensors, vol. 15, no. 12, pp. 30 240-30260, 2015. 
[38] W. Yi, M. Jiang, S. Li, and B. Wang, "Distributed sensor fusion for RFS density with consideration of limited sensing ability," in International Conference on Information Fusion (Fusion), 2017, pp. 1-6.

[39] B.-N. Vo and W. K. Ma, "The Gaussian Mixture Probability Hypothesis Density Filter," IEEE Transactions on Signal Processing, vol. 54, no. 11, pp. 4091-4104, 2006.

[40] P. F. Felzenszwalb, R. B. Girshick, D. McAllester, and D. Ramanan, "Object Detection with Discriminatively Trained Part-Based Models," IEEE Transactions on Pattern Analysis and Machine Intelligence, vol. 32, no. 9, pp. 1627-1645, 2010.

[41] K. Granstrom, M. Baum, and S. Reuter, "Extended object tracking: Introduction, overview and applications," Journal of Advances in Information Fusion, vol. 12, no. 2, pp. 139 - 174, 2016.

[42] E. Insafutdinov, L. Pishchulin, B. Andres, M. Andriluka, and B. Schiele, "DeeperCut: A Deeper, Stronger, and Faster Multi-person Pose Estimation Model," in European Conference on Computer Vision (ECCV), 2016, pp. 34-50.

[43] H. W. Kuhn, The Hungarian method for the assignment problem. Naval Research Logistics Quarterly, 1955.

[44] B. Ristic, D. Clark, B. N. Vo, and B. T. Vo, "Adaptive Target Birth Intensity for PHD and CPHD Filters," IEEE Transactions on Aerospace and Electronic Systems, vol. 48, no. 2, pp. 1656-1668, 2012.

[45] B.-N. Vo, S. Singh, and D. Arnaud, "Sequential Monte Carlo methods for multitarget filtering with random finite sets," IEEE Transactions on Aerospace and Electronic Systems, vol. 51, no. 4, pp. 1224-1245, 2005.

[46] M. Vasic, D. Mansolino, and A. Martinoli, "A system implementation and evaluation of a cooperative fusion and tracking algorithm based on a gaussian mixture phd filter," in IEEE/RSJ International Conference on Intelligent Robots and Systems (IROS), 2016, pp. 4172-4179.

[47] M. Danelljan, G. Hger, F. S. Khan, and M. Felsberg, "Convolutional Features for Correlation Filter Based Visual Tracking," in IEEE International Conference on Computer Vision Workshop (ICCVW), 2015, pp. $621-629$.

[48] A. Milan, L. Leal-Taixe, I. Reid, S. Roth, and K. Schindler, "MOT16: A Benchmark for Multi-Object Tracking," arXiv:1603.00831 [cs.CV], pp. 1-13, 2016.

[49] K. Bernardin and R. Stiefelhagen, "Evaluating Multiple Object Tracking Performance: The CLEAR MOT Metrics," EURASIP Journal on Image and Video Processing, vol. 2008, pp. 1-10, 2008.

[50] M. Vasic and A. Martinoli, "A Collaborative Sensor Fusion Algorithm for Multi-object Tracking Using a Gaussian Mixture Probability Hypothesis Density Filter," in IEEE International Conference on Intelligent Transportation Systems, 2015, pp. 491-498.

[51] N. Wojke and A. Bewley, "Deep cosine metric learning for person re-identification," in 2018 IEEE Winter Conference on Applications of Computer Vision (WACV), 2018, pp. 748-756.

[52] S. H. Bae and K. J. Yoon, "Confidence-Based Data Association and Discriminative Deep Appearance Learning for Robust Online MultiObject Tracking," IEEE Transactions on Pattern Analysis and Machine Intelligence, vol. 40, no. 3, pp. 595-610, 2018.

[53] M. Keuper, S. Tang, Y. Zhongjie, B. Andres, T. Brox, and B. Schiele, "A multi-cut Formulation for Joint Segmentation and Tracking of Multiple Objects," arXiv:1607.06317 [cs.CV], pp. 1-18, 2016.

[54] C. Kim, F. Li, A. Ciptadi, and J. M. Rehg, "Multiple Hypothesis Tracking Revisited," in IEEE International Conference on Computer Vision (ICCV), 2015, pp. 4696-4704.

[55] J. Chen, H. Sheng, Y. Zhang, and Z. Xiong, "Enhancing Detection Model for Multiple Hypothesis Tracking," in IEEE Conference on Computer Vision and Pattern Recognition Workshops (CVPRW), 2017, pp. 21432152.

[56] J. Son, M. Baek, M. Cho, and B. Han, "Multi-Object Tracking with Quadruplet Convolutional Neural Networks," in IEEE Conference on Computer Vision and Pattern Recognition (CVPR), 2017, pp. 56205629.

[57] W. Wang, J. Shen, and L. Shao, "Video salient object detection via fully convolutional networks," IEEE Transactions on Image Processing, vol. 27 , no. 1, pp. 38-49, 2018.

[58] W. Wang and J. Shen, "Deep visual attention prediction," IEEE Transactions on Image Processing, vol. 27, no. 5, pp. 2368-2378, 2018.

[59] X. Dong, J. Shen, D. Yu, W. Wang, J. Liu, and H. Huang, "Occlusionaware real-time object tracking," IEEE Transactions on Multimedia, vol. 19, no. 4, pp. 763-771, 2017.

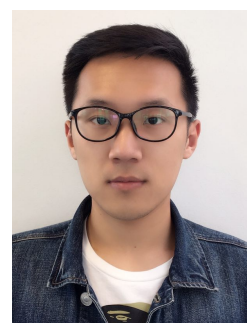

Zeyu Fu (S'15-M'18) received the B.Eng. degree in Electrical and Electronic Engineering with 1stclass Honours from Newcastle University, Newcastle Upon Tyne, U.K., in 2015 . He is currently pursuing the Ph.D. degree with Intelligent Sensing and Communications (ISC) Research Group, School of Engineering, Newcastle University, U.K

His research areas of interest include video based multi-target tracking, enhanced RFS filtering, dictionary learning, deep learning.

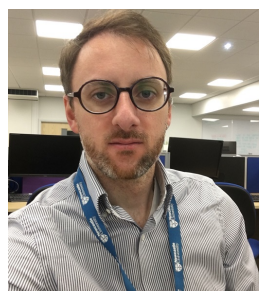

Federico Angelini, (S'17) received the Master degree in Pure and Applied Mathematics from University of Rome "Tor Vergata", Italy, in 2015 . He collaborated with National Research Council - CNR, Rome, Italy on Image Denoising Methods project in 2015-2016. He is currently pursuing the Ph.D. degree with Intelligent Sensing and Communications (ISC) Research Group, School of Engineering, Newcastle University, U.K.

His research interests include Human Action and Behaviour Recognition via Multimodal Sensors, Machine Learning and Signal Processing.

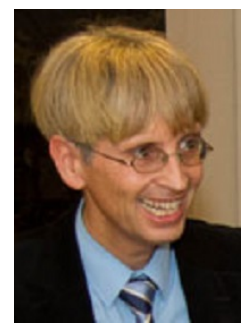

Jonathon Chambers (S'83-M'90-SM'98-F'11) received the Ph.D. and D.Sc. degrees in signal processing from the Imperial College of Science, Technology and Medicine (Imperial College London), London, U.K., in 1990 and 2014, respectively. On 1st Dec 2017 he became the Head of the Engineering Department at the University of Leicester. He is also an International Honorary Dean and Guest Professor within the Department of Automation at Harbin Engineering University, China. His research interests include adaptive signal processing and machine learning and their application in communications, defence and navigation systems.

Dr. Chambers is a Fellow of the Royal Academy of Engineering, U.K., the Institution of Engineering and Technology, and the Institute of Mathematics and its Applications. He has served as an Associate Editor for the IEEE TRANSACTIONS ON SIGNAL PROCESSING for three terms over the periods 1997-1999, 2004-2007, and as a Senior Area Editor 2011-2015.

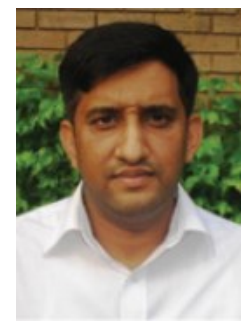

Syed Mohsen Naqvi (S'07-M'09-SM'14) received the Ph.D. degree in Signal Processing from Loughborough University, Loughborough, U.K., in 2009 and his Ph.D. thesis was on the EPSRC U.K. funded project. He was a Postdoctoral Research Associate on the EPSRC U.K.-funded projects and REF Lecturer from 2009 to 2015. Prior to his postgraduate studies in Cardiff and Loughborough Universities U.K., he served the National Engineering and Scientific Commission (NESCOM) of Pakistan from Jan 2002 to Sep 2005.

Dr Naqvi is a Lecturer in Signal and Information Processing at the School of Engineering, Newcastle University, Newcastle, U.K. He has 100+ publications with the main focus of his research being on Multimodal (audio-video) Signal and Information Processing. He is Fellow of the Higher Education Academy (FHEA). His research interests include multimodal processing for human behaviour analysis, multi-target tracking, and source separation all; for machine learning. He organized special sessions on multi-target tracking in FUSION 2013\&2014, delivered seminars and was a speaker at UDRC Summer School 2015-2017. 